\title{
COMPUTERS IN BIOLOGY AND MEDICINE
}

https://www.journals.elsevier.com/computers-in-biology-and-medicine/editorial-board/edward-ciaccio-phd

Editor in Chief: Prof. Dr. Edward Ciaccio, Columbia University, New York, USA.

Impact Factor: 3.434; ISSN: 0010-4825

(ACCEPTED JANUARY $4^{\text {TH } 2021)}$

\section{COMPUTATIONAL SIMULATIONS OF HYBRID MEDIATED NANO- HEMODYNAMICS (AG-AU/BLOOD) THROUGH AN IRREGULAR SYMMETRIC STENOSIS}

\author{
Jayati Tripathi' ${ }^{1}$ B. Vasu ${ }^{1}$ * and $O$. Anwar Bég ${ }^{2}$ \\ ${ }^{1}$ Department of Mathematics, Motilal Nehru National Institute of Technology Allahabad, \\ Prayagraj, Uttar Pradesh- 211004, India. \\ ${ }^{2}$ Professor and Director-Multi-Physical Engineering Sciences Group (MPESG), Department of \\ Mechanical and Aeronautical Engineering, School of Science, Engineering and Environment \\ (SEE), Newton Building-G77/G84, Salford University, The Crescent, Manchester, M54WT, UK.
}

\section{ABSTRACT:}

*Corresponding author-email: bvasu@mnnit.ac.in

This article examines theoretically and numerically the unsteady two-dimensional blood flow through a diseased artery featuring an irregular stenosis. An appropriate geometric model is adopted to simulate the irregular stenotic artery. Inspired by drug delivery applications for blood vessels, the impact of hybrid nanoparticles on blood flow using a modified Tiwari-Das model is discussed. The blood is examined to have a homogenous suspension of hybrid nanoparticles. Reynolds' viscosity model is applied in the formulation to represent the temperature dependency of blood. The two-dimensional governing conservation equations for momentum and heat transfer with buoyancy effect are simplified by considering the mild stenotic approximation. A finite-difference technique is deployed to numerically discretize the transformed non-dimensional model. Extensive graphical results for blood flow characteristics are obtained by MATLAB code. Comprehensive visualization of the effects of hemodynamic, geometric and nanoscale parameters on transport characteristics is provided. The problem is conducted for silver and silver-gold hybrid mediated blood flow models, and experimental values of blood and these biocompatible metallic nanoparticles. A comparison between silver and hybrid nanofluid is obtained which promotes the use of hybrid nanoparticles in successfully achieving clinically more beneficial results associated with nano-drug delivery in diseased hemodynamics. Enhancement in viscosity parameter induces axial flow acceleration in the stenotic region while lower thermal conductivity decreases the temperature magnitudes. Furthermore, with time variation, the pressure gradient is found to be lower in coronary arteries comparatively to femoral arteries. The simulations are relevant to transport phenomenon in nano-drug targeted delivery in haematology.

KEYWORDS: Arterial stenosis; Unsteady viscous hemodynamics; Hybrid nanoparticles (Ag-Au/Blood); Nano-drug delivery; Finite Difference Method (FDM); Reynolds's model; heat transfer; thermal buoyancy; wall shear stress (WSS). 


\section{NOMENCLATURE}

$B_{1} \quad$ Pressure gradient parameter $(\mathrm{Pa} / \mathrm{m})$

d Distance of stenosis from origin (m)

$l_{o} \quad$ Length of stenosis (m)

Gr Grashof number (-)

Pr Prandtl number (-)

p Pressure $(\mathrm{Pa})$

$Q \quad$ Flow rate $\left(\mathrm{m}^{3} / \mathrm{s}\right)$

$r \quad$ Radial co-ordinate $(\mathrm{m})$

$R \quad$ Radius of the artery (stenotic) (m)

$R_{0} \quad$ Radius of the artery (non-stenotic) (m)

Re Reynolds number (-)

$t \quad$ Time (s)

$T$ Temperature (K)

$T_{1} \quad$ Reference temperature (K)

$T_{w} \quad$ Wall temperature $(\mathrm{K})$

$u \quad$ Radial velocity $(\mathrm{m} / \mathrm{s})$

$U_{o} \quad$ Reference velocity $(\mathrm{m} / \mathrm{s})$

$w \quad$ Axial velocity $(\mathrm{m} / \mathrm{s})$

$z \quad$ axial co-ordinate $(\mathrm{m})$

Greek Letters

$\theta \quad$ Dimensionless temperature (-)

$\mu_{0} \quad$ Reference viscosity $(\mathrm{kg} /(\mathrm{ms}))$

$\mu \quad \operatorname{Viscosity~}(\mathrm{kg} /(\mathrm{ms}))$

$\rho$ Density $\left(\mathrm{kg} / \mathrm{m}^{3}\right)$

$\phi \quad$ Nanoparticle concentration (Moles $/ \mathrm{m}^{3}$ )

$C_{p} \quad$ Heat capacitance $(\mathrm{J} / \mathrm{kgK})$

$k \quad$ Thermal conductivity $(\mathrm{W} / \mathrm{kgK})$ 
$\eta_{0} \quad$ Viscosity constant (-)

$\delta \quad$ Depth of stenosis (m)

$\gamma \quad$ Thermal expansion coefficient $(/ \mathrm{K})$

$\tau \quad$ Wall shear stress $\left(\mathrm{N} / \mathrm{m}^{2}\right)$

\section{INTRODUCTION}

Blood flow has an extensive role in the generation of cardiovascular diseases. In recent years, mathematical modeling and computational simulation in hemodynamics have been extensively explored to improve the understanding of causes of distinct diseases and identify improved modern treatments for mitigation or regulation of these ailments. Atherosclerosis is the most common arterial disease in which fat deposits inside the wall of the artery. This phenomenon reduces the area of the lumen, which is usually known as stenosis [1]. Stenosis formation obstructs the blood flow rate and reduces the supply of oxygen and nutrients to different parts of the body, a condition clinically termed as ischemia. In coronary arteries, ischemia may account for myocardial infarction, while in the brain, it may precipitate a stroke. Hence the generation of stenosis can quickly deteriorate into a life-threatening situation. Blood has a complex macroscopic behavior due to the properties of the constituents and the composition of blood. Blood having a shear rate greater than $100 \mathrm{~s}^{-1}$ generally shows a Newtonian nature. However, for a shear rate of less than $100 \mathrm{~s}^{-1}$, blood is observed to be non-Newtonian in nature. As cardiovascular diseases contribute to a majority of deaths around the globe, therefore more elegant hemodynamic models that furnish a more accurate representation of hematological transport are becoming increasingly critical in combating this situation. As the stenosis does not have any specific shapes and there are various geometries of stenosis (composite, overlapped, bell shaped, irregular etc.) based on the different data. As mostly studies have assumed that the stenosis could be represented by smooth mathematical function, but in reality, stenoses contain many valleys and ridges similar to mountain ranges. With the experimental data, Johnston and Kilpatrick [2] considered the irregular stenosis for understanding blood flow characteristics. Chakravarty and Mandal [3] developed a blood flow model for stenosed arterial flow, considering an overlapping stenosis and indicating that blood velocity reduces in the stenosis downstream but increases in the upstream region. Mustapha et al. [4] utilized a MAC (Marker and Cell) method and successive-over-relaxation (SOR) with 
staggered grids to solve the pressure-Poisson equation with pressure-velocity correction formulae in unsteady multi-irregular stenosed arterial hemodynamics. Likewise, Abdullah et al. [5] used an optimized finite difference code to simulate transient hydromagnetic micropolar rheological blood flow through an irregular stenosis. Furthermore, Ellahi et al. [6] conducted a theoretical study for slip blood flow through an arterial segment under the composite stenosis and considering blood as non-Newtonian micropolar fluid. They derived analytic solutions of velocity and volumetric flow flux using modified Bessel functions, observing significant flow pattern modifications are induced with stenosis, and the tapering effect and flow impedance is elevated with increasing the stenosis height. Zaman et al. [7] presented an axisymmetric model for blood flow through a tapered catheterized artery containing both an aneurysm and stenosis using the Eringen non-Newtonian micropolar fluid model. They computed gyration (micro-rotation) and velocity distributions with a finite difference scheme and observed significant deviation from the Newtonian case with increasing micropolar vortex viscosity effects.

The statistics emphasize the urgent requirement for new and more effective therapies and technologies which can prevent these diseases. The existing treatments for CVDs aim to restore the normal blood flow around the damaged vasculature and prevent recurring phenomenon. Banach et al. [8] suggested that by statin therapy, reduction in build-up and thickening of plaques can be achieved. However, such therapies also have adverse side effects and poor patient compliance, which suggests that modifications of such treatments are needed. This among other factors has accelerated the deployment of nanotechnology (the leading area in the 21st century sciences globally) in biomedicine and witnessed the birth of a new field - nanomedicine. In particular, stenotic nano-hemodynamics has witnessed considerable interest in the engineering sciences and applied mathematics communities owing to its significance in combating diseased arteries. The many studies reported have also featured numerous computational methods which are required to tackle the nonlinearity of the mathematical models. In 1995, Choi [9] introduced "nanofluids," which constitute colloidal suspensions of nanometer-sized particles (1-100nm) in a base fluid. These particles may be carbides, oxides, metallic or non-metallic and the base fluids include water, mineral oil, ethylene glycol, etc. It has been extensively verified that the inclusion of nanoparticles in the base fluid increases the thermal conductivity by a remarkable degree. To understand the heat transfer phenomenon by suspending the small (nano) particles in fluid is basically given by two approaches: single-phase model and two-phase model. The single-phase 
model is applicable when both the fluid phase and solid particles are flow with same local velocity and are in thermal equilibrium state. However in two-phase model, both the solid and fluid have different phases in heat transfer process. The nanoparticles can be easily fluidized and as a result, the nanofluid can almost be considered as a conventional single-phase (homogenous) fluid with physical properties of individual phases. The main assumptions that we consider in this model are: the slip between the nanoparticles and the base fluid is negligible and there is always a thermal and hydrodynamic equilibrium in solid and fluid phases. Nanoparticles are also synthetic materials in some applications related to biomedicine due to the unique way of interacting with matter [10]. They may also be biodegradable and biocompatible for human safety. Among numerous nanotechnology applications, targeted drug delivery (nano-pharmacodynamics) has been explored widely as a mechanism for enabling clinicians to bring significant improvement at a molecular level to the field of healthcare. In drug delivery systems, using large size of materials poses major challenges, which includes poor bioavailability and solubility, issue with target specificity, in vivo stability and tonic effectiveness with probable side effects. Therefore, in advanced biomedicine, nanotechnology plays an interesting role in drug formulations which control drug release and deliver the drugs with more specificity. Nanoparticles are usually small sized nanospheres, hence in comparison to bigger material they can move more freely in human body. Nowadays nanomedicine has become more valuable because they could be utilised as delivery agents by attaching therapeutic drugs to it and then delivers it to targeted area with controlled release. Tripathi and Bég [11] reported one of the earliest theoretical studies of nanofluids for biomedical applications. Likewise, Tripathi et al. [12] conducted a review of recent advancements in hemodynamic aspects of nano-drug delivery systems. Vasu et al. [13] later developed a robust finite element model for non-Newtonian blood flow conveying nanoparticles in a stenosed coronary artery using FREEFEM++ software and the Buongiorno two-component nanoscale model. Ahmed and Nadeem [14] examined the performance of three distinct nanoparticles $(\mathrm{Cu}$, $\mathrm{Al} 2 \mathrm{O} 3$, and $\mathrm{TiO} 2)$ in blood flow through a stenosed artery. Dubey et al. [15] very recently developed detailed numerical solutions and visualizations of the non-Newtonian blood flow containing nanoparticles through an artery having a combination of stenosis and aneurysm. Tariq et al. [16] analyzed the theoretical study of incompressible viscous fluid having inhomogeneous dust particles. Results exhibited that on increasing the slip parameter, velocity of both fluid and dust is decreased. Zaman and Khan [17] studied the unsteady non-Newtonian (Carreau) blood flow 
in w-shaped stenosed artery. In stenosis channel, curvature effects are taken into account and the impact of nanoparticles on various profiles is discussed.

In recent years biomedical researchers have also scrutinized the possibilities of multiple nanoparticles in nanofluids. Makishima et al. [18] suggested suspensions of two or more metals in base fluid to provide a homogenous phase, termed the hybrid nanofluid. Compared to unitary nanofluids (i.e., base fluids with a single nanoparticle species), hybrid nanofluids combine the physical and chemical properties of different nanoparticles, which are proven to be more potent in medical applications. The hybrid nanofluids have significant potential in the fields of drug delivery, gene therapy, hyperthermia, and MRI treatments. Ijaz and Nadeem [19] theoretically investigated the steady-state hybrid nanoparticles mediated blood flow through an overlapped stenosed artery, including tapering effects. The hemodynamics in a stenosed artery doped with hybrid (silver, copper, and gold) nanoparticles has also been considered by Ijaz and Nadeem [20]. Their study revealed that among all the other nanoparticles, gold nanoparticles have a more astonishing impact, although they provided somewhat limited visualizations of the flow regime. Zaman et al. [21] analyzed the blood flow characteristics of a curved stenosed artery with an aneurysm. Hybrid nanoparticles (copper, silver) were considered, and the emerging non-linear partial differential equations were solved using the FTCS method.

Generally, blood viscosity is considered constant in hemodynamic computations, although this assumption is not practical for actual cardiovascular transport. Viscosity is found to be a function of temperature and pressure in the case of real fluids, including blood. For some years, many investigations have been performed considering viscosity as a function of temperature, such as Reynolds' model, Vogel's model, Einstein's model, etc. Zaman et al. [22] used the Reynolds' model to represent the viscosity of blood for biomedical simulations of hybrid nanofluids in a stenosed artery. Ellahi et al. [23] used two different viscosity models (Vogel's and Reynolds' model) for fully developed annular nanofluid flows, deriving Homotopy power series solutions.

The article has been motivated by applications in targeted nano-drug delivery system. The potential application of numerical blood flow simulation is to aid decision making processes during the treatment of cardiovascular diseases. Although a conventional method for treating the stenosis is deploying a stent or catheter inside the artery, but nowadays targeting the nano-drugs at desired location is increasingly trending. This also triggers the process of clotting formation at stenosis 
throat and the effect of these post-treatment processes can also be predicted by computational simulation. With the motivation of probing deeper into Newtonian pharmacodynamics transport in realistic physiological geometries, the current article examines the characteristics of hybrid nanoparticles (i.e., silver and gold, Ag, Au) in unsteady blood flow theoretically through irregular symmetric stenosis in a diseased artery. In addition to this, the pulsatile pressure gradient and Reynolds viscosity model are also considered. This research's main novelty is the combination of hybrid nanoparticles $(\mathrm{Ag}, \mathrm{Au})$ with variable viscosity effects, which to the author's knowledge has thus far not received attention in computational biomedical fluid dynamics. A modified TiwariDas nanoscale model is used, enabling unique properties to be determined for actual nanoparticles (viscosity, thermal conductivity, specific heat capacity, density, etc.). The normalized non-linear conservation equations are solved by a robust finite difference method, and further simulations and computations are performed on MATLAB. The current study is divided into the following sections: Section 1 contains the literature review. Section 2 illustrates the arterial geometry of the problem (for irregular stenosis). Section 3 explains the mathematical formulation of the hybrid nanofluid transport model. Section 4 describes the non-dimensionalization of the model equations. The explanation of the numerical technique is described in Section 5. Validation is done in section 6. Further, results and discussion for hemodynamic transport characteristics (velocity, temperature, wall shear stress, and flow rate) are elaborated in Section 7. Lastly, the main conclusions of the study are summarized in Section 8.

\section{GEOMETRY OF THE MODEL}

In this study, unsteady, incompressible, and laminar blood flow through an axisymmetric artery, which has a diseased segment as irregular stenosis, is investigated. The mathematical equation representing the geometry of the artery [24] is formulated in a cylindrical coordinate system $(r, \theta, z)$ as:

$$
R(z)=\left\{\begin{array}{ll}
R_{o}-2 \delta\left[\cos \left(2 \pi\left(\frac{z-d}{2}-\frac{l_{o}}{4}\right)\right)-\frac{7}{100} \cos \left(32 \pi\left(z-d-\frac{l_{o}}{2}\right)\right)\right], & d<z<d+l_{o} \\
R_{o}, & \text { otherwise. }
\end{array}\right\}
$$


In the above expression $R_{o}$ denotes the radius of normal artery, $\delta$ denotes the height of stenosis and $d$ is the distance of stenosis from origin. The geometry of the considered artery is given by Figure 1 with suspended nanoparticles and blood cells.

\section{MATHEMATICAL HYBRID NANOPARTICLE TRANSPORT MODEL}

Considering the blood flowing through the artery is bi-directional, the velocity and temperature fields can be presented as:

$V=[u(r, z, t), 0, w(r, z, t)], \quad T=T(r, z, t)$.

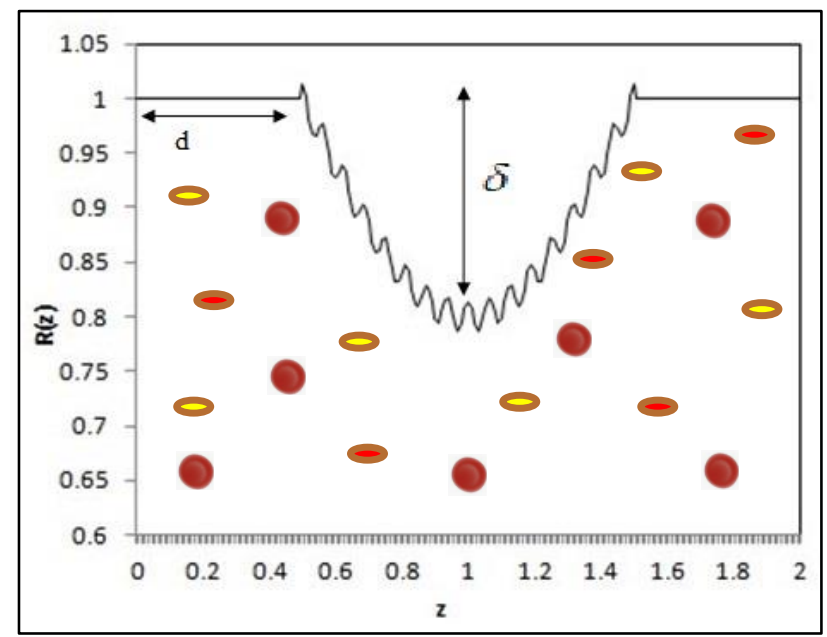

- Blood cell

- Nanoparticle

Figure 1: Geometry of the stenotic arterial segment in which $\mathrm{R}(\mathrm{z})$ is radius of artery, $\mathrm{z}$ is axial direction while $\delta$ shows the depth of stenosis

In Eq. (2), the radial and the axial velocity components are denoted by $u$ and $w$ respectively. Therefore, the equation of continuity, momentum, and energy for hybrid nanofluids (blood doped with hybrid nanoparticles) are expressed as follows [25]:

$\frac{\partial u}{\partial r}+\frac{u}{r}+\frac{\partial w}{\partial z}=0$

$\rho_{h n f}\left[\frac{\partial u}{\partial t}+u \frac{\partial u}{\partial r}+w \frac{\partial u}{\partial z}\right]=-\frac{\partial p}{\partial r}+\frac{1}{r} \frac{\partial}{\partial r}\left[\mu_{h n f}(T) \frac{\partial u}{\partial r}\right]+\frac{\partial}{\partial z}\left[\mu_{h n f}(T)\left(\frac{\partial u}{\partial z}+\frac{\partial w}{\partial r}\right)\right]-2 \mu_{h n f}(T) \frac{u}{r^{2}}$ 


$$
\begin{aligned}
& \rho_{h n f}\left[\frac{\partial w}{\partial t}+u \frac{\partial w}{\partial r}+w \frac{\partial w}{\partial z}\right]=-\frac{\partial p}{\partial z}+\frac{1}{r} \frac{\partial}{\partial r}\left[r \mu_{h n f}(T)\left(\frac{\partial u}{\partial z}+\frac{\partial w}{\partial r}\right)\right]+\frac{\partial}{\partial z}\left[2 \mu_{h n f}(T)\left(\frac{\partial w}{\partial z}\right)\right]+(\rho \gamma)_{h n f} g\left(T-T_{1}\right), \\
& \left(\rho C_{p}\right)_{h n f}\left[\frac{\partial T}{\partial t}+u \frac{\partial T}{\partial r}+w \frac{\partial T}{\partial z}\right]=k_{h n f}\left[\frac{\partial^{2} T}{\partial r^{2}}+\frac{1}{r} \frac{\partial T}{\partial r}+\frac{\partial^{2} T}{\partial z^{2}}\right] .
\end{aligned}
$$

The physical parameters for the hybrid nano-fluid featuring in the above equations are as follows: $\mu_{h n f}$ is viscosity, $\rho_{h n f}$ is density, $k_{h n f}$ is thermal conductivity, $\gamma_{h n f}$ is thermal expansion and $\left(\rho C_{p}\right)_{h n f}$ is heat capacitance respectively. The corresponding boundary and initial conditions are prescribed by Eq. (7)-(8) and a figure of boundary conditions in stenosed vessel with nanoparticles in given in Figure 2.

Boundary Conditions: $\left.\quad \frac{\partial w}{\partial r}\right|_{r=0}=0,\left.\quad w\right|_{r=R}=0,\left.\quad \frac{\partial T}{\partial r}\right|_{r=0}=0,\left.\quad T\right|_{r=R}=1$,

Initial Conditions: $\left.\quad w\right|_{t=0}=0,\left.\quad T\right|_{t=0}=0$.

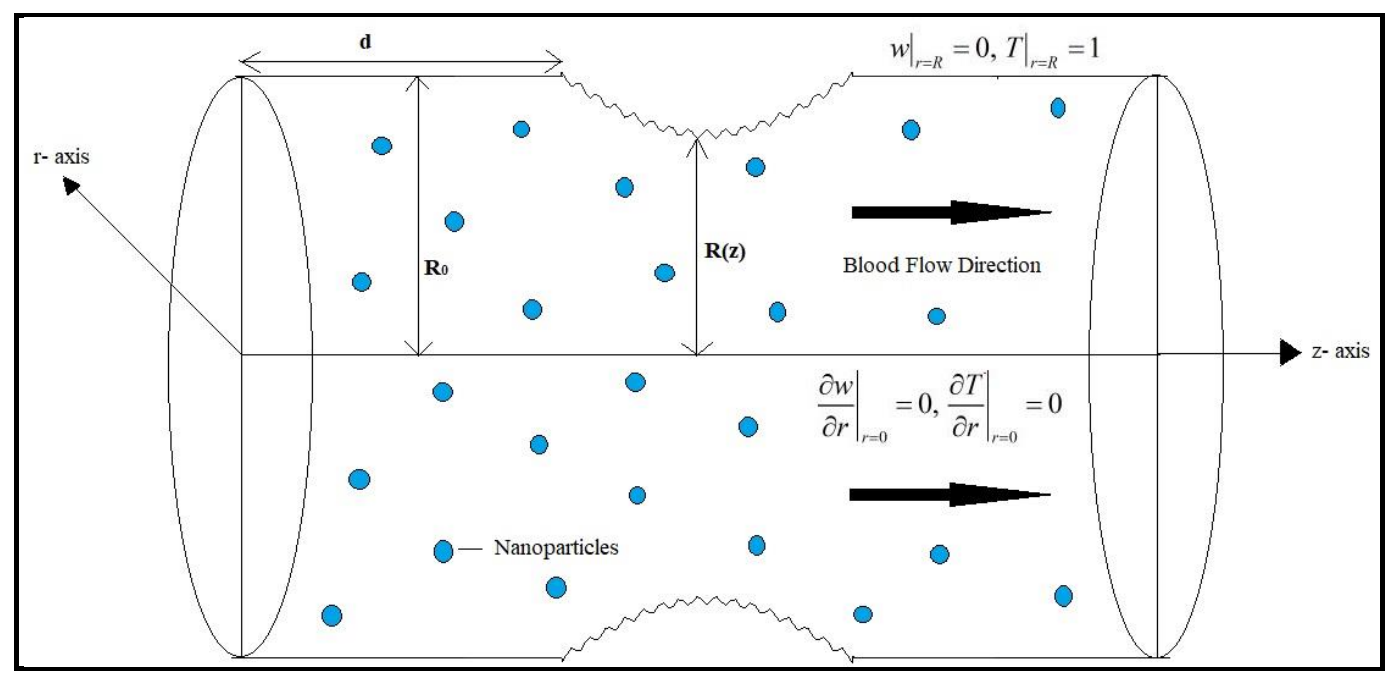

Figure 2: Representation of boundary conditions in the stenosed artery

\section{NON-DIMENSIONALIZATION OF HYBRID NANOFLUID MODEL}

In this section, to facilitate a numerical solution and enable scaling of fluid dynamic phenomena, the above governing Equations (3)-(6) are non-dimensionalized with the help of the following transformation variables [25] given by Eq. (9): 


$$
\begin{aligned}
& \bar{r}=\frac{r}{R_{o}}, \quad \bar{w}=\frac{w}{R_{o}}, \quad \bar{u}=\frac{l_{o} u}{\delta^{*} U_{o}}, \quad \bar{t}=\frac{U_{o} t}{R_{o}}, \quad \bar{z}=\frac{z}{l_{o}}, \quad \bar{p}=\frac{R_{o}^{2} P}{U_{o} l_{o} \mu_{o}}, \quad \theta=\frac{\left(T-T_{1}\right)}{\left(T_{w}-T_{1}\right)} \\
& \bar{R}=\frac{R}{R_{o}}, \quad \operatorname{Pr}=\frac{C_{p} \mu_{f}}{k_{f}}, \quad \operatorname{Re}=\frac{U_{o} \rho_{f} R_{o}}{\mu_{f}}, \quad G r=\frac{\rho_{f} R_{o}^{2} g \gamma_{f}\left(T_{w}-T_{1}\right)}{\mu_{f} U_{o}}, \quad \bar{d}=\frac{d}{l_{0}} .
\end{aligned}
$$

Here $R e$ is Reynolds number, $\operatorname{Pr}$ is Prandtl number, $G r$ is Grashof number, $T_{w}$ is wall temperature and $U_{o}$ denotes reference velocity. In the above-defined variables, the non-dimensional geometric parameters are stenosis height parameter $\left(\delta=\delta^{*} / R_{0}\right)$ and vessel aspect $\operatorname{ratio}\left(\varepsilon=R_{0} / l_{o}\right)$. Introducing the above-defined variables and after dropping the bars, Equations (3)-(6) will take the form:

$\delta\left[\frac{\partial u}{\partial r}+\frac{u}{r}\right]+\frac{\partial w}{\partial z}=0$

$$
\begin{array}{r}
\operatorname{Re}\left(\frac{\rho_{h n f}}{\rho_{f}}\right) \delta \varepsilon^{2}\left[\frac{\partial u}{\partial t}+(\delta \varepsilon) u \frac{\partial u}{\partial r}+\varepsilon w \frac{\partial u}{\partial z}\right]=-\left(\frac{\partial p}{\partial r}\right)+\left(\frac{\delta R_{o}}{l_{o}^{2} \mu_{o}}\right) \frac{1}{r} \frac{\partial}{\partial r}\left[\mu_{h n f}(\theta) \frac{\partial u}{\partial r}\right]+ \\
\left(\frac{\varepsilon^{2} R_{o}}{\mu_{o}}\right) \frac{\partial}{\partial z}\left[\mu_{h n f}(\theta)\left(\frac{\delta \varepsilon}{l_{o}} \frac{\partial u}{\partial z}+\frac{1}{R_{o}} \frac{\partial w}{\partial r}\right)\right]-2\left(\frac{\delta \varepsilon^{2}}{\mu_{o}}\right) \mu_{h n f}(\theta)\left(\frac{u}{r^{2}}\right),
\end{array}
$$

$$
\begin{aligned}
\operatorname{Re}\left(\frac{\rho_{h n f}}{\rho_{f}}\right)\left[\frac{\partial w}{\partial t}+(\delta \varepsilon) u \frac{\partial w}{\partial r}+\varepsilon w \frac{\partial w}{\partial z}\right]= & -\left(\frac{\partial p}{\partial z}\right)+\frac{1}{r} \frac{\partial}{\partial r}\left[\frac{r \mu_{h n f}(\theta)}{\mu_{o}}\left\{\delta \varepsilon^{2} \frac{\partial u}{\partial z}+\frac{\partial w}{\partial r}\right\}\right]+ \\
& \varepsilon^{2} \frac{\partial}{\partial z}\left[\frac{2 \mu_{h n f}(\theta)}{\mu_{o}} \frac{\partial w}{\partial z}\right]+\frac{(\rho \gamma)_{h n f}}{(\rho \gamma)_{f}}(G r) \theta
\end{aligned}
$$

$\operatorname{Re} \operatorname{Pr}\left(\frac{\left(\rho C_{p}\right)_{h n f}}{\left(\rho C_{p}\right)_{f}}\right)\left(\frac{k_{f}}{k_{h n f}}\right)\left[\frac{\partial \theta}{\partial t}+(\delta \varepsilon) u \frac{\partial \theta}{\partial r}+\varepsilon w \frac{\partial \theta}{\partial z}\right]=\left[\frac{\partial^{2} \theta}{\partial r^{2}}+\frac{1}{r} \frac{\partial \theta}{\partial r}+\varepsilon^{2} \frac{\partial^{2} \theta}{\partial z^{2}}\right]$

Here for the formulation of the hybrid nanofluid viscosity, the exponential Reynolds viscosity model [23] is utilized which may be expressed is:

$\mu_{f}(\theta)=\mu_{o} e^{-\eta_{o} \theta}=\mu_{o}\left[1-\eta_{o} \theta\right]$, where $\eta_{o} \ll 1$. 
Here the parameter $\eta_{o}$ is a viscosity constant. The equations for the thermophysical parameters of the nanofluid are represented by [26]:

$$
\begin{aligned}
& \mu_{n f}(\theta)=\frac{\mu_{f}(\theta)}{(1-\phi)^{5 / 2}}, \quad \rho_{n f}=(1-\phi) \rho_{f}+\phi \rho_{s}, \\
& \left(\rho C_{p}\right)_{n f}=(1-\phi)\left(\rho C_{p}\right)_{f}+\phi\left(\rho C_{p}\right)_{s}, \quad(\rho \gamma)_{n f}=(1-\phi)(\rho \gamma)_{f}+\phi(\rho \gamma)_{s}, \\
& \frac{k_{n f}}{k_{f}}=\frac{k_{s}+2 k_{f}-2 \phi\left(k_{f}-k_{s}\right)}{k_{s}+2 k_{f}+\phi\left(k_{f}-k_{s}\right)} .
\end{aligned}
$$

Additionally, the equations for thermo-physical properties of hybrid mediated nano-fluid assume the forms [26]:

$$
\begin{aligned}
& \mu_{h n f}=\frac{\mu_{f}(\theta)}{\left(1-\phi_{1}\right)^{5 / 2}\left(1-\phi_{2}\right)^{5 / 2}}, \quad \rho_{h n f}=\left(1-\phi_{2}\right)\left[\left(1-\phi_{1}\right) \rho_{f}+\phi_{1} \rho_{S_{1}}\right]+\phi_{2} \rho_{S_{2}}, \\
& \left(\rho C_{p}\right)_{h n f}=\left(1-\phi_{2}\right)\left[\left(1-\phi_{1}\right)\left(\rho C_{p}\right)_{f}+\phi_{1}\left(\rho C_{p}\right)_{S_{1}}\right]+\phi_{2}\left(\rho C_{p}\right)_{S_{2}}, \\
& (\rho \gamma)_{h n f}=\left(1-\phi_{2}\right)\left[\left(1-\phi_{1}\right)(\rho \gamma)_{f}+\phi_{1}(\rho \gamma)_{S_{1}}\right]+\phi_{2}(\rho \gamma)_{S_{2}}, \\
& \frac{k_{h n f}}{k_{b f}}=\frac{k_{S_{2}}+2 k_{b f}-2 \phi_{2}\left(k_{b f}-k_{S_{2}}\right)}{k_{S_{2}}+2 k_{b f}+\phi_{2}\left(k_{b f}-k_{S_{2}}\right)}, \quad \text { where } \frac{k_{b f}}{k_{f}}=\frac{k_{S_{1}}+2 k_{f}-2 \phi_{1}\left(k_{f}-k_{S_{1}}\right)}{k_{S_{1}}+2 k_{f}+\phi_{1}\left(k_{f}-k_{S_{1}}\right)} .
\end{aligned}
$$

\begin{tabular}{|c|c|c|c|c|c|c|}
\hline \multirow[t]{2}{*}{ Thermophysical properties } & \multirow{2}{*}{$\begin{array}{l}\text { Blood } \\
\text { (subscript,f) }\end{array}$} & \multicolumn{5}{|c|}{ Metallic Nanoparticles (subscript, s) } \\
\hline & & $(\mathrm{Ag})$ & $(\mathrm{Au})$ & $(\mathrm{Cu})$ & $(\mathrm{TiO} 2)$ & $(\mathrm{SiO} 2)$ \\
\hline $\operatorname{Density}\left[\rho\left(k g / m^{3}\right)\right]$ & 1063 & 10500 & 19320 & 8933 & 4250 & 2200 \\
\hline $\begin{array}{l}\text { Thermal Conductivity } \\
{[K(W / m K)]}\end{array}$ & 0.492 & 429 & 314 & 400 & 8.9538 & 1.4 \\
\hline $\begin{array}{l}\text { Thermal expansion } \\
\text { coefficient }\left[\gamma \times \mathbf{1 0}^{-5}\left(K^{-1}\right)\right]\end{array}$ & 0.18 & 1.8 & 1.4 & 1.67 & 0.9 & 42.7 \\
\hline $\begin{array}{ll}\text { Heat } & \text { Capacitance } \\
{\left[C_{p}(J / k g K)\right]} & \end{array}$ & 3594 & 235 & 129 & 385 & 686.2 & 745 \\
\hline
\end{tabular}

Table 1: Thermophysical properties of blood and nanoparticles 
In the above-mentioned equations, $\mu_{f}$ is the viscosity of the base fluid, $\rho_{f}$ as density, $\left(\rho C_{p}\right)_{f}$ is heat capacitance, $\gamma_{f}$ is thermal expansion coefficient and $k_{f}$ is the thermal conductivity of the base fluid. For different nanoparticles, $\left(\phi_{1}, \phi_{2}\right)$ stands for the volume fraction, $\left(\rho_{S_{1}}, \rho_{S_{2}}\right)$ is the density of solid particles, $\left[\left(\rho C_{p}\right)_{S_{1}},\left(\rho C_{p}\right)_{S_{2}}\right]$ is the heat capacitance, $\left[\gamma_{S_{1}}, \gamma_{S_{2}}\right]$ is a thermal expansion coefficient and $\left[k_{S_{1}}, k_{S_{2}}\right]$ is thermal conductivity of solid nanoparticles, respectively. For the blood and nanoparticles Silver $(A g)$ and Gold $(A u)$, thermo-physical properties are given in Table 1.

Consequently, non-dimensionalized equations have been modified with two hypotheses $\delta \ll 1$ and $\varepsilon=O(1)$ i.e., the maximum height of stenosis is lesser compared with the radius of the artery and also that the radius of the artery and length of the stenotic region are of comparable magnitude. After imposing these suppositions, Equations (10)-(13) contract to:

$$
\begin{aligned}
& \frac{\partial w}{\partial z}=0 \\
& \frac{\partial p}{\partial r}=0
\end{aligned}
$$

$\operatorname{Re}\left(\frac{\rho_{h n f}}{\rho_{f}}\right)\left[\frac{\partial w}{\partial t}\right]=-\frac{\partial p}{\partial z}+\frac{1}{r} \frac{\partial}{\partial r}\left[r\left(\frac{\mu_{h n f}(\theta)}{\mu_{o}}\right)\left(\frac{\partial w}{\partial r}\right)\right]+\frac{(\rho \gamma)_{h n f}}{(\rho \gamma)_{f}} G r \theta$

$$
\frac{\left(\rho C_{p}\right)_{h n f}}{\left(\rho C_{p}\right)_{f}}\left(\frac{k_{f}}{k_{h n f}}\right) \operatorname{Pr} \operatorname{Re}\left[\frac{\partial \theta}{\partial t}\right]=\left[\frac{\partial^{2} \theta}{\partial r^{2}}+\frac{1}{r} \frac{\partial \theta}{\partial r}\right]
$$

Subsequently, with the help of Burton [27], the axial pressure gradient can be represented as:

$$
-\frac{\partial p}{\partial z}=A_{o}+A_{1} \cos \left(2 \pi w_{p}\right) t, t>0
$$

By using the non-dimensionalized parameters defined in Eq. (9), the normalized form of the pressure gradient becomes:

$$
-\frac{\partial p}{\partial z}=B_{1}\left[1+e \cos \left(c_{1} t\right)\right]
$$


Where $e=\frac{A_{1}}{A_{o}}, \quad B_{1}=\frac{A_{o} a^{2}}{\mu_{o} U_{o}}, \quad c_{1}=\frac{2 \pi a w_{p}}{U_{o}}$.

Invoking Eq. (21) into the axial momentum equation, Eq. (18) emerges as:

$\operatorname{Re}\left(\frac{\rho_{h n f}}{\rho_{f}}\right)\left[\frac{\partial w}{\partial t}\right]=B_{1}\left[1+e \cos \left(c_{1} t\right)\right]+\frac{1}{r} \frac{\partial}{\partial r}\left[r\left(\frac{\mu_{h n f}(\theta)}{\mu_{o}}\right)\left(\frac{\partial w}{\partial r}\right)\right]+\frac{(\rho \gamma)_{h n f}}{(\rho \gamma)_{f}} G r \theta$

Further, Equations (23) and (19) are subject to following boundary and initial conditions:

$$
\begin{array}{lll}
\left.w(r, t)\right|_{r=R}=0, & \left.\frac{\partial w(r, t)}{\partial r}\right|_{r=0}=0, & w(r, 0)=0, \\
\left.\theta(r, t)\right|_{r=R}=1, & \left.\frac{\partial \theta(r, t)}{\partial r}\right|_{r=0}=0, & \theta(r, 0)=0 .
\end{array}
$$

The mathematical quantities such as volumetric flow rate, wall shear stress (WSS) and resistance impedance may be formulated as:

$$
\begin{aligned}
& Q_{1}=2 \pi \int_{0}^{R} w r d r \\
& \tau_{s}=-\left(\frac{\partial w}{\partial r}\right)_{r=R}, \\
& \lambda=\frac{L\left(\frac{\partial p}{\partial z}\right)}{Q} .
\end{aligned}
$$

With respect to the above non-dimensional parameters i.e. Eq. (9), the geometrical Eq. (1) in dimensionless form can be written as:

$$
R(z)=\left\{\begin{array}{ll}
1-2 \delta^{*}\left[\cos \left(2 \pi\left(\frac{z-d}{2}-\frac{1}{4}\right)\right)-\frac{7}{100} \cos \left(32 \pi\left(z-d-\frac{1}{2}\right)\right)\right], & d<\bar{z}<d+1, \\
1, & \text { otherwise. }
\end{array}\right\}
$$

Further the transformed Equations (23) and (19) are simplified using radial coordinate 
transformation $\left(x=\frac{r}{R(z)}\right)$, which leads to the following set of partial differential equations with associated boundary and initial conditions:

$$
\begin{aligned}
& \left(\frac{\rho_{h n f}}{\rho_{f}}\right) \operatorname{Re}\left[\frac{\partial w}{\partial t}\right]=B_{1}\left[1+e \cos \left(c_{1} t\right)\right]+\left(\frac{\mu_{h n f}(\theta)}{\mu_{o}}\right)\left(\frac{1}{R^{2}}\right)\left[\frac{\partial^{2} w}{\partial x^{2}}+\frac{1}{x} \frac{\partial w}{\partial x}\right]+\frac{(\rho \gamma)_{h n f}}{(\rho \gamma)_{f}} G r \theta \\
& \left(\frac{\left(\rho C_{p}\right)_{h n f}}{\left(\rho C_{p}\right)_{f}}\right)\left(\frac{k_{f}}{k_{h n f}}\right) \operatorname{Pr} \operatorname{Re}\left[\frac{\partial \theta}{\partial t}\right]=\left(\frac{1}{R^{2}}\right)\left[\frac{\partial^{2} \theta}{\partial x^{2}}+\frac{1}{x} \frac{\partial \theta}{\partial x}\right] .
\end{aligned}
$$

Furthermore, the associated dimensionless boundary conditions (24)-(25) are written as:

$$
\begin{array}{ll}
\left.\frac{\partial w}{\partial x}\right|_{x=0}=0, & \left.w\right|_{x=1}=0,\left.\quad \frac{\partial \theta}{\partial x}\right|_{x=0}=0,\left.\quad \theta\right|_{x=1}=1 \\
\left.w\right|_{t=0}=0, & \left.\theta\right|_{t=0}=0 .
\end{array}
$$

Similarly, the volumetric flow rate, wall shear stress (WSS) and impedance respectively [Equations (26)-(28)] take the following form:

$$
\begin{aligned}
& Q_{1}=2 \pi R^{2}\left(\int_{0}^{1} w x d x\right) \\
& \tau_{s}=-\frac{1}{R}\left(\frac{\partial w}{\partial x}\right)_{x=1}, \\
& \lambda=\frac{L\left(\frac{\partial p}{\partial z}\right)}{Q}
\end{aligned}
$$

Replacing the pressure gradient term in Eq. (36), it can be written:

$$
\lambda=\frac{L\left[B_{1}(1+e \cos (2 \pi t))\right]}{2 \pi R^{2}\left(\int_{0}^{1} w x d x\right)}
$$


Introducing the values of thermophysical properties of hybrid nanofluid in Equations (30) - (31), the momentum and energy equations will be reduced to:

$$
\begin{aligned}
& {\left[\left(1-\phi_{2}\right)\left[\left(1-\phi_{1}\right)+\phi_{1} \frac{\rho_{S_{1}}}{\rho_{f}}\right]+\phi_{2} \frac{\rho_{S_{2}}}{\rho_{f}}\right] \operatorname{Re}\left[\frac{\partial w}{\partial t}\right]=B_{1}\left[1+e \cos \left(c_{1} t\right)\right]+\frac{\left(1-\eta_{o} \theta\right)}{\left(1-\phi_{1}\right)^{5 / 2}\left(1-\phi_{2}\right)^{5 / 2}}\left(\frac{1}{R^{2}}\right)\left[\frac{\partial^{2} w}{\partial x^{2}}+\frac{1}{x} \frac{\partial w}{\partial x}\right]+} \\
& {\left[\left(1-\phi_{2}\right)\left[\left(1-\phi_{1}\right)+\phi_{1} \frac{(\rho \gamma)_{S_{1}}}{(\rho \gamma)_{f}}\right]+\phi_{2} \frac{(\rho \gamma)_{S_{2}}}{(\rho \gamma)_{f}}\right] \operatorname{Gr} \theta} \\
& {\left[\left(1-\phi_{2}\right)\left[\left(1-\phi_{1}\right)+\phi_{1} \frac{\left(\rho C_{p}\right)_{S_{1}}}{\left(\rho C_{p}\right)_{f}}\right]+\phi_{2} \frac{\left(\rho C_{p}\right)_{S_{2}}}{\left(\rho C_{p}\right)_{f}}\right]\left(\frac{k_{f}}{k_{h n f}}\right) \operatorname{Pr} \operatorname{Re}\left[\frac{\partial \theta}{\partial t}\right]=\left(\frac{1}{R^{2}}\right)\left[\frac{\partial^{2} \theta}{\partial x^{2}}+\frac{1}{x} \frac{\partial \theta}{\partial x}\right]}
\end{aligned}
$$

\section{NUMERICAL SOLUTION}

The exact solution of the nonlinear partial differential equations (38)-(39) is difficult, if not intractable. Hence it is necessary to use numerical methods for solving the equations defined mathematically by Equations (38)-(39). A robust, suitable numerical technique based on the discretization of partial differential equations is the explicit finite-difference technique, which is described in detail by Hoffmann [28]. Furthermore, this scheme is also known as FTCS, i.e., forward in time (FT) and central in space (CS) and has been implemented by various researchers in many diverse studies [21, 25]. In this scheme, firstly, the spatial domain is discretized, and after that, the value of velocity component is calculated at each node $x_{i}$ and over the time instant $t^{k}$, which is written as $w_{i}^{k}$. According to Hoffmann [28], the central differencing formulation for second order and the forward differencing formulation for first-order partial derivatives are defined as:

$$
\begin{aligned}
& \frac{\partial w}{\partial x} \cong \frac{w_{i+1}^{k}-w_{i-1}^{k}}{2 \Delta x}=w_{x}, \quad \frac{\partial^{2} w}{\partial x^{2}} \cong \frac{w_{i+1}^{k}-2 w_{i}^{k}+w_{i-1}^{k}}{(\Delta x)^{2}}=w_{x x} \\
& \text { and } \frac{\partial w}{\partial t} \cong \frac{w_{i}^{k+1}-w_{i}^{k}}{(\Delta t)}
\end{aligned}
$$


Incorporating the values of the described partial derivatives, Equations (38)-(39) will readily be reduced to the following forms:

$$
\begin{aligned}
& w_{i}^{k+1}=w_{i}^{k}+\frac{\Delta t}{\operatorname{Re}\left[\left(1-\phi_{2}\right)\left[\left(1-\phi_{1}\right)+\phi_{1} \frac{\rho_{S_{1}}}{\rho_{f}}\right]+\phi_{2} \frac{\rho_{S_{2}}}{\rho_{f}}\right]}\left[\begin{array}{c}
B_{1}\left[1+e \cos \left(c_{1} t^{k}\right)\right]+\frac{\left(1-\eta_{o} \theta_{i}^{k}\right)}{\left(1-\phi_{1}\right)^{5 / 2}\left(1-\phi_{2}\right)^{5 / 2}}\left(\frac{1}{R^{2}}\right)\left[\frac{\partial^{2} w}{\partial x^{2}}+\frac{1}{x} \frac{\partial w}{\partial x}\right]+ \\
{\left[\left(1-\phi_{2}\right)\left[\left(1-\phi_{1}\right)+\phi_{1} \frac{(\rho \gamma)_{S_{1}}}{(\rho \gamma)_{f}}\right]+\phi_{2} \frac{(\rho \gamma)_{S_{2}}}{(\rho \gamma)_{f}}\right] G r \theta_{i}^{k}}
\end{array}\right], \\
& \theta_{i}^{k+1}=\theta_{i}^{k}+\left(\frac{k_{h n f}}{k_{f}}\right) \frac{\Delta t}{\operatorname{Pr} \operatorname{Re}\left[\left(1-\phi_{2}\right)\left[\left(1-\phi_{1}\right)+\phi_{1} \frac{\left(\rho C_{p}\right)_{S_{1}}}{\left(\rho C_{p}\right)_{f}}\right]+\phi_{2} \frac{\left(\rho C_{p}\right)_{S_{2}}}{\left(\rho C_{p}\right)_{f}}\right]}\left\{\left(\frac{1}{R^{2}}\right)\left[\frac{\partial^{2} \theta}{\partial x^{2}}+\frac{1}{x} \frac{\partial \theta}{\partial x}\right]\right\},
\end{aligned}
$$

Further, the associated boundary and initial conditions (32)-(33) are represented by:

$$
\begin{array}{ll}
w_{i}^{1}=\theta_{i}^{1}=0 \quad \text { at } t=0, & w_{i+1}^{k}=w_{i}^{k}, \theta_{i+1}^{k}=\theta_{i}^{k} \text { at } \quad x=0, \\
w_{N+1}^{k}=0, \quad \theta_{N+1}^{k}=1, \quad & \text { at } \quad x=1 .
\end{array}
$$

In this numerical scheme, the spatial variable is discretized into $N+1$ grid points, where the step size is taken as $\Delta x=1 / N+1$. The time instant is defined by $t_{k}$, where the value of $t_{k}$ is given as $t_{k}=(k-1) \Delta t$, in which $\Delta t$ designates a small increment in time. The velocity component is further calculated at each node and for every time instant. As stability of this numerical scheme is dependent on step size and time increment, hence $\Delta x=0.025$ and $\Delta t=0.0001$ are carefully selected to fulfill the stability condition. These values confirm the stability and convergence of this scheme, as also verified in Hoffmann [28]. The FTCS code has been extensively validated and implemented in numerous previous simulations, including solar energy radiative heat transfer flows [29], periodic hydromagnetic flows in geophysics [30], viscoelastic blood flow with body acceleration [31], peristaltic gastro-intestinal pumping [32], thermosolutal Cross fluid blood flow in tapered arteries [33] and non-Newtonian coating hydromechanics [34]. Confidence is, therefore, justifiably high in the FTCS scheme. 


\section{VALIDATION OF THE NUMERICAL RESULTS:}

Thank you for pointing this out. To validate the numerical method which has been chosen to solve the current problem, the numerical results obtained by Zaman et al. [25] for the axial velocity profile of blood flow are compared with present results which also have been computed by using the same modelling with FTCS method and shown in Table 2. From the table, the comparison states that the results are in good agreement with each other.

\begin{tabular}{|c|c|c|c|c|}
\hline $\mathrm{R}$ & $\begin{array}{c}\text { Zaman et al. [25] } \\
w_{S}=0.0\end{array}$ & $\begin{array}{c}\text { Present Results } \\
w_{S}=0.0\end{array}$ & $\begin{array}{c}\text { Zaman et al. [25] } \\
w_{S}=0.1\end{array}$ & $\begin{array}{c}\text { Present Results } \\
w_{S}=0.1\end{array}$ \\
\hline 0 & 0.5859 & 0.5881 & 0.6857 & 0.6833 \\
\hline 0.10 & 0.5829 & 0.5845 & 0.6827 & 0.6798 \\
\hline 0.20 & 0.5725 & 0.5725 & 0.6723 & 0.6680 \\
\hline 0.30 & 0.5540 & 0.5518 & 0.6539 & 0.6476 \\
\hline 0.40 & 0.5261 & 0.5215 & 0.6259 & 0.6177 \\
\hline 0.50 & 0.4864 & 0.4802 & 0.5863 & 0.5770 \\
\hline 0.60 & 0.4323 & 0.4257 & 0.5322 & 0.5231 \\
\hline 0.70 & 0.3605 & 0.3549 & 0.4604 & 0.4529 \\
\hline 0.80 & 0.2671 & 0.2637 & 0.3671 & 0.3624 \\
\hline 0.90 & 0.1483 & 0.1473 & 0.2483 & 0.2466 \\
\hline 1.00 & 0 & 0 & 0.1000 & 0.1000 \\
\hline
\end{tabular}

Table 2: Comparison of results with Zaman et al. [25] for axial velocity results at throat of stenotic artery $z=0.71$ and $t=1.15$.

\section{RESULTS AND DISCUSSION:}

In this section, extensive results of numerical FTCS computations are provided. In particular, the modification in hemodynamics of irregular symmetric stenotic arterial transport in the presence of hybrid nanoparticles is discussed. Concerning the emerging featured thermophysical and hemodynamic parameters, their impact on axial velocity, temperature profile, wall shear stress, volumetric flow rate, and pressure gradient are visualized graphically. Additionally, to illustrate the influence of hybrid nanofluids (i.e., Ag-Au/Blood) in comparison to uni-nanofluids (i.e., 
$\mathrm{Ag} / \mathrm{Blood}$ ), different graphical results are presented with the help of the considered momentum and energy equations. In the numerical computations with the FTCS code, Table 3 represents the default values of all parameters used. The data is consistent with stenotic blood flows and nanoparticle drug delivery $[35,36]$.

\begin{tabular}{|l|l|l|l|l|l|l|l|l|}
\hline Parameter & $\phi_{1}$ & $\phi_{2}$ & $B_{1}$ & $c_{1}$ & $\eta_{0}$ & $G r$ & $\operatorname{Pr}$ & $\operatorname{Re}$ \\
\hline Value & 0.03 & 0.03 & 1.41 & 1 & 0.2 & 0.1 & 14 & 2 \\
\hline
\end{tabular}

Table 3: Default value of emerging parameters

Figures 3 (a)-(b) display the effect of different volume fractions of silver and hybrid nanoparticles $\left(\phi_{1}, \phi_{2}\right)$ on the axial velocity profile at the stenotic throat of the artery. Figure 3(a) shows only the impact of silver nanoparticles $\left(\phi_{1}\right)$ on velocity, whereas figure 3 (b) illustrates the effect of both the volume fractions $\left(\phi_{1}, \phi_{2}\right)$. From both the figures, it is noted that the velocity profile is a decreasing function for the volume fraction parameter. On comparing figure 3 (b) with figure 3 (a), it is evident that the addition of a second nanoparticle type increases the decrement in velocity value, and this result is hugely favorable for arterial diseases to control blood flow.
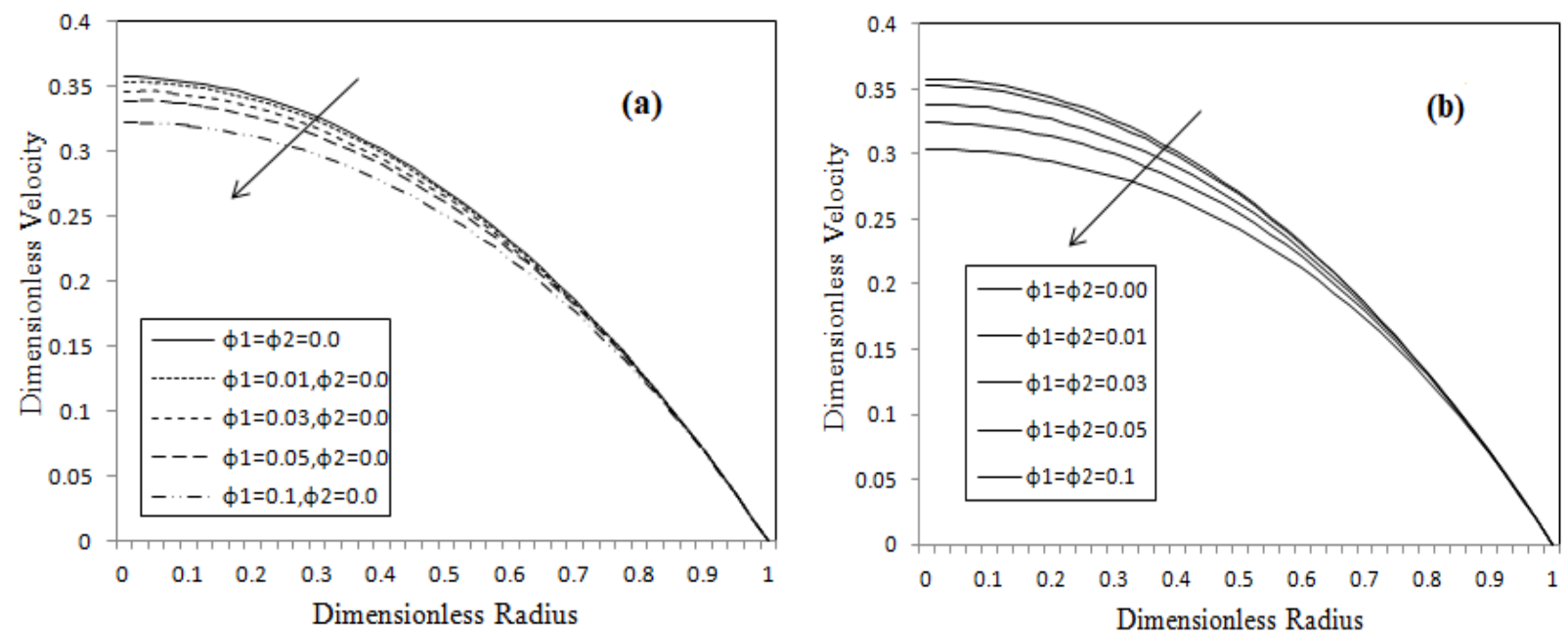

Figure 3: Effect of nanoparticle concentration on axial velocity with (a) Ag nanofluid only (b) Ag-Au hybrid nanofluid for $B_{1}=1.41, \delta=0.1, z=0.98, t=1.2$ 

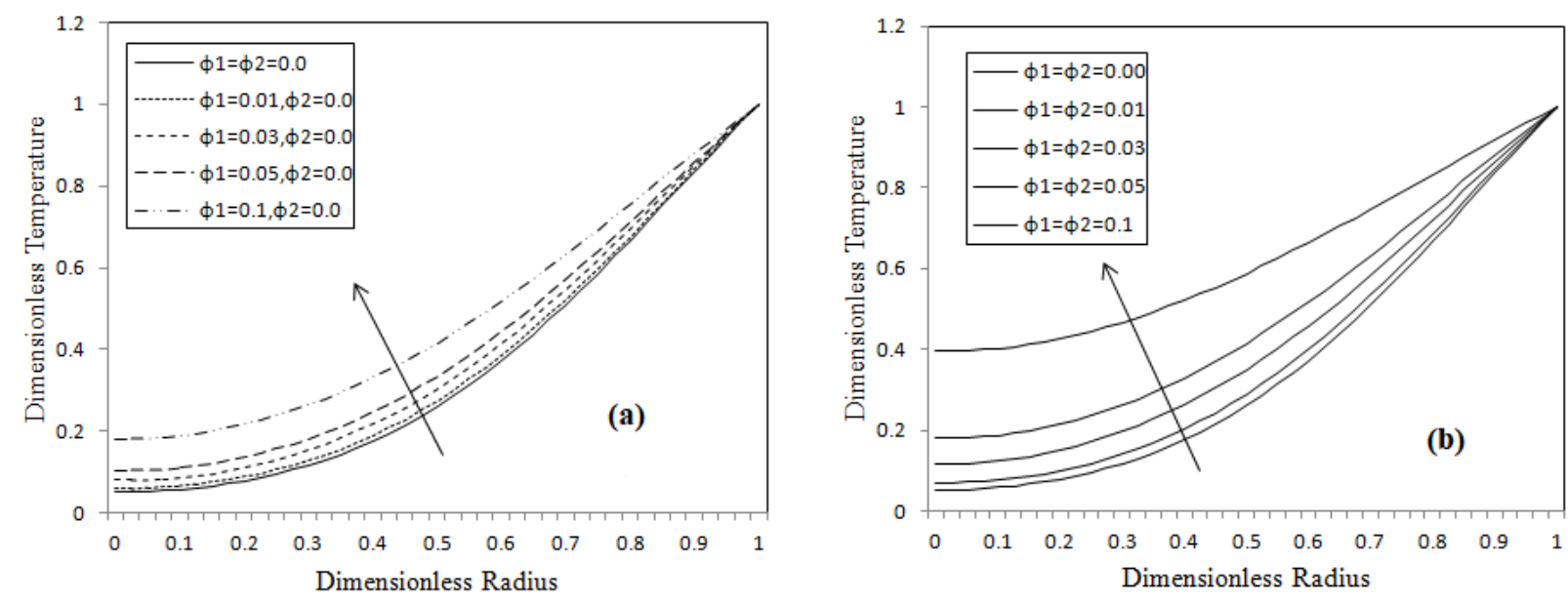

Figure 4: Effect of nanoparticle concentration on temperature profile with (a) Ag nanofluid only (b) Ag-Au hybrid nanofluid for $B_{1}=1.41, \delta=0.1, z=0.98, t=1.2$.

Figure 4 depicts the temperature profile response to different nanoparticle concentrations for (a) Ag nanofluid only (b) Ag-Au hybrid nanofluid. A significant temperature elevation is computed with an increment in nanoparticle volume fraction, i.e., temperatures are minimized in the absence of nanoparticles. Significantly greater temperatures are also generated with the hybrid case (Fig. 4b) compared to the unitary nanofluid case (Fig. 4a). The implication is that the amalgamation of nanoparticles in blood enhances thermal diffusion in the stenotic vessel and assists in the transport process.
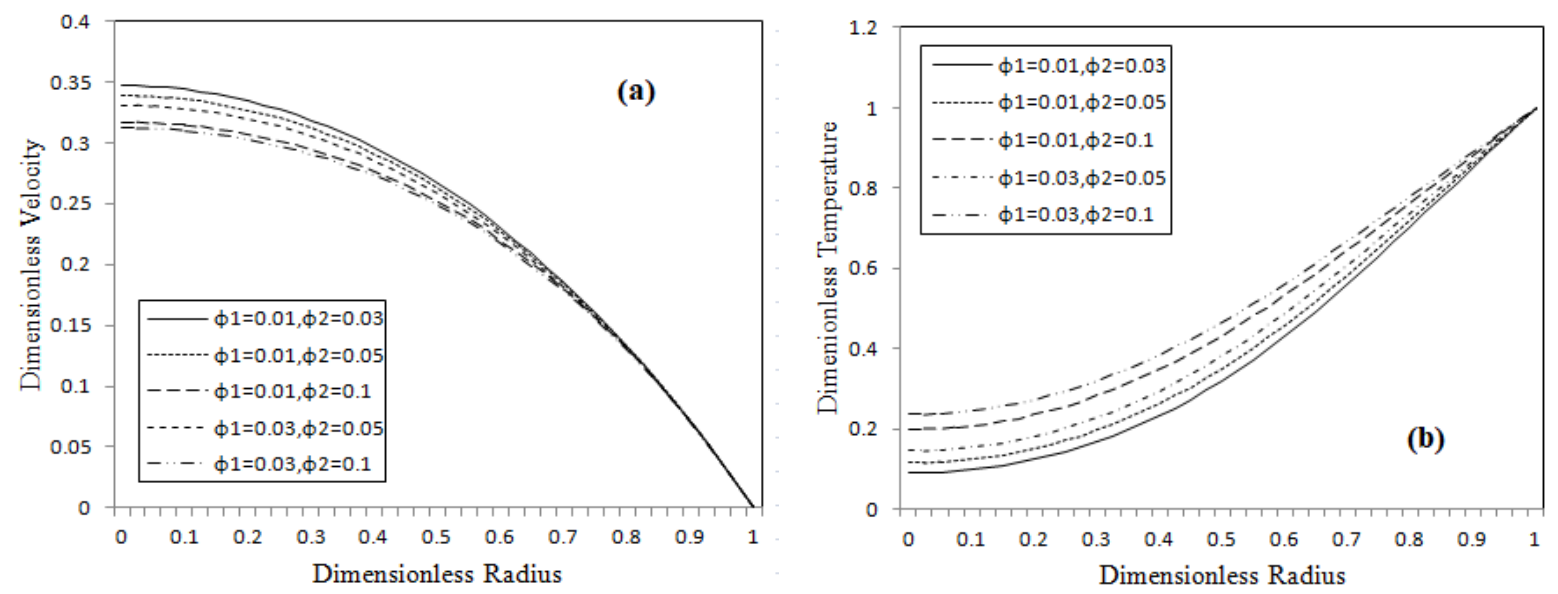

Figure 5: Effect of different combination of hybrid nanoparticles on (a) velocity and (b) temperature profile for

$$
B_{1}=1.41, \delta=0.1, z=0.98, t=1.2 \text {. }
$$


Figure 5 illustrates the velocity and temperature evolution with different combinations of hybrid nanoparticles $\left(\phi_{1}, \phi_{2}\right)$. Inspection of figure 5 (a) indicates that a pattern is noted on changing both nanoparticle concentrations $\phi_{1}$ (silver) and $\phi_{2}$ (gold) from 0.01 to 0.1 , i.e., a significant amount of reduction can be seen. Among all the various combinations of nanoparticle concentrations, the most significant decrement in axial velocity is computed for $\phi_{1}=0.03$ and $\phi_{2}=0.1$. Similarly, from figure 5 (b), it is observed that temperature magnitudes are accentuated with increasing value of volume fraction of both nanoparticles. Similar to the velocity profile, the nanoparticle concentrations $\phi_{1}=0.03$ and $\phi_{2}=0.1$ also induce the most tremendous change in temperature values.
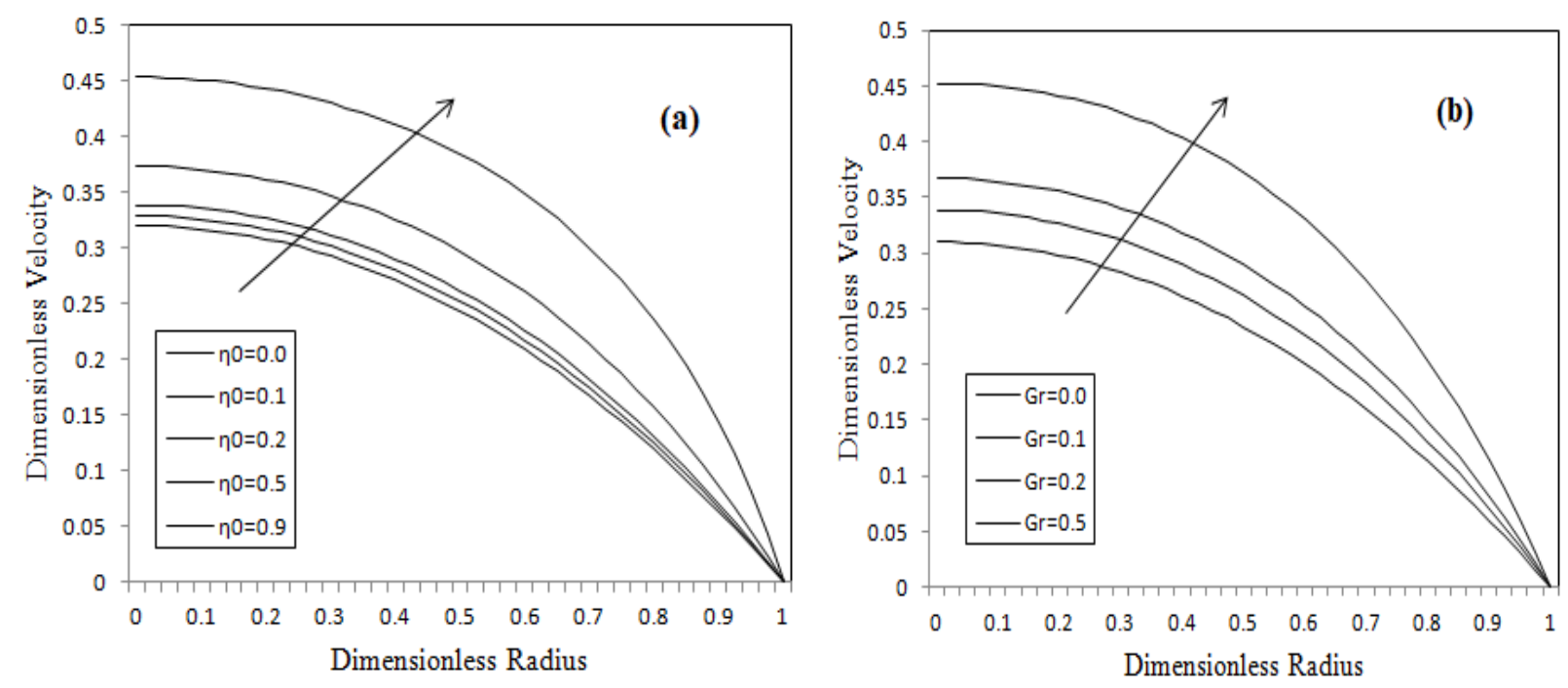

Figure 6: Effect of (a) $\eta_{0}$ and (b) Grashof number on axial velocity for following data

$$
B_{1}=1.41, \delta=0.1, z=0.98, t=1.2
$$

The velocity profiles for different nanofluid viscosity, $\eta_{0}$ and Grashof number, $G r$ are plotted in Figures 6 (a)-(b). These figures exhibit a similar pattern for both the emerging parameters $\left(\eta_{0}, G r\right)$ i.e., by increasing the value of any of the parameters $\eta_{0}$ or $G r$, the magnitude of the axial velocity is also increased. In other words, greater nanofluid viscosity and thermal buoyancy effect manifest in axial flow acceleration. In figure 6 (a), the range of nano-fluid viscosity is taken as 0.0-0.9, while for figure 6(b), the Grashof number is taken in the range of 0.0-0.5. The acceleration in velocity implies that the blood viscosity or resistance among the particles has clearly reduced with 
higher magnitudes of the parameter $\left(\eta_{0}\right)$. Further, the Grashof number $(\mathrm{Gr})$ is the ratio of buoyancy force to viscous force. Since the nanoparticle species diffusion is influenced by the concentration differences of nanoparticles within blood increases the thermal buoyancy force. This phenomenon further leads to a reduction in resistance in the blood flow and a concomitant acceleration. Evidently, flow deceleration arises with vanishing thermal buoyancy force $(G r=0)$ as seen in Figure $6 b$.

Figures 7 (a)-(b) portrays the influence of parameters $B_{1}$ and $c_{1}$ appearing in the dimensionless form of the pressure gradient equation. For different sizes of an artery, e.g., arterioles, coronary artery, the value of $B_{1}$ is 1.41 , whereas for the femoral artery of the human body, it changes to 6.6, at a particular location known as the 'throat' of the stenotic artery. Figure 7 (a) indicates that on increasing the value of the $B_{1}$ parameter, the blood velocity experiences strong acceleration. This implies that in large (femoral) arteries, velocity is higher compared to smaller (coronary) arteries. The effect of the $c_{1}$ parameter on the velocity profile is illustrated in Figure 7 (b). It is noticed that at the time $t=1.2$, velocity decreases significantly with an increasing value of $c_{1}$ from 0.5 to 3 , i.e., again hemodynamic retardation is induced.
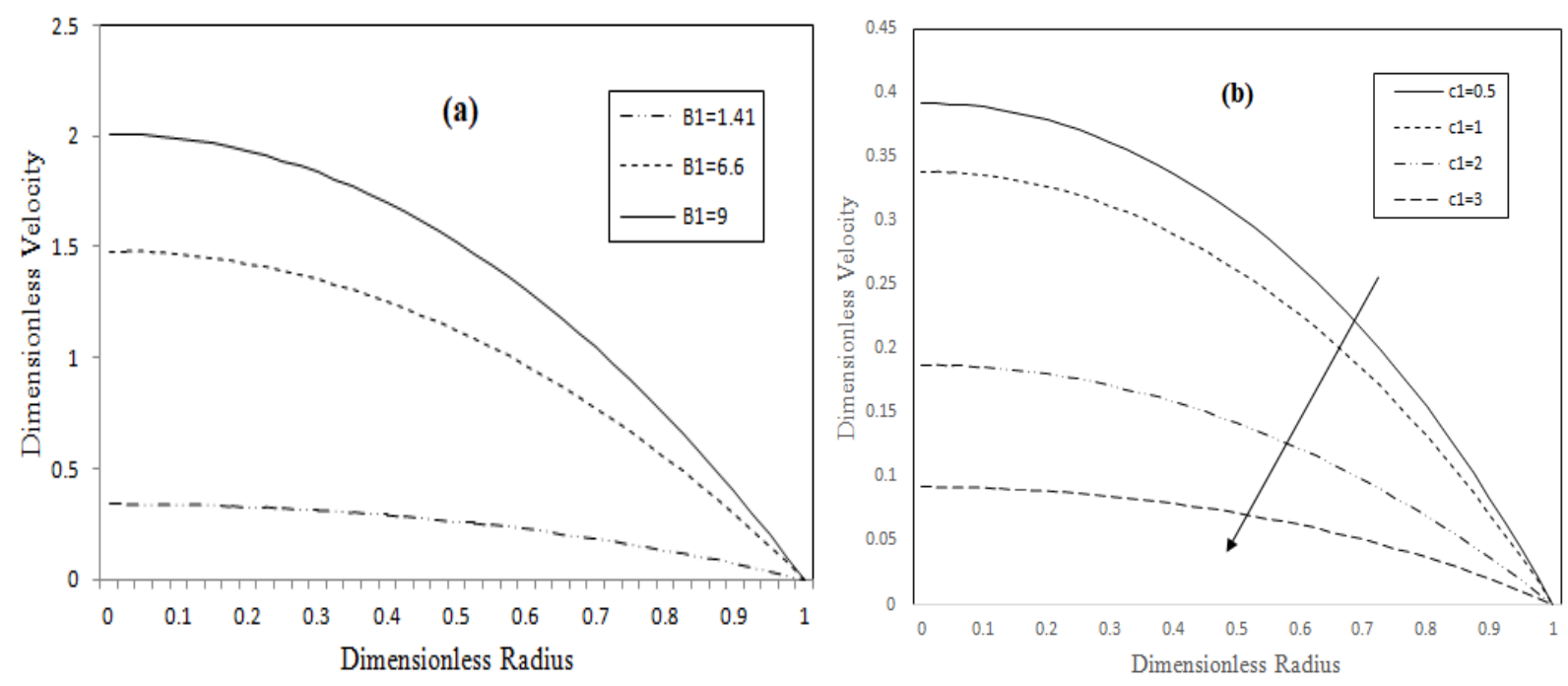

Figure 7: Effect on velocity profile for different (a) $B 1$ parameter (b) $c_{l}$ parameter for $\delta=0.1, z=0.98, t=1.2$

Figure 8 (a)-(b) shows the response in velocity and temperature of the blood to the Prandtl number $(\mathrm{Pr})$. From both the figures, it is apparent that temperatures are suppressed with the increasing value of $P r$. Prandtl number is the single most important parameter in thermal transport in fluids 
and is a property of a particular fluid under specific conditions. It embodies the ratio of momentum diffusivity to thermal diffusivity and, as such, is inversely proportional to the thermal conductivity of the fluid. Higher thermal conductivity fluids have lower Prandtl numbers and vice versa. When $\operatorname{Pr}=1$, both the momentum and thermal diffusion rate are equivalent, and velocity and thermal boundary layers at the arterial walls will be of the same thickness. $\operatorname{Pr}$ also has an inverse relationship with the heat transfer phenomenon from the artery wall to the fluid. When $\operatorname{Pr}$ is significantly small, the diffusion of heat significantly exceeds the momentum diffusivity. The curve trends in Figure 8 (a)-(b) confirm that the higher the Prandtl number, the lower the hemodynamic velocity and temperature magnitude.
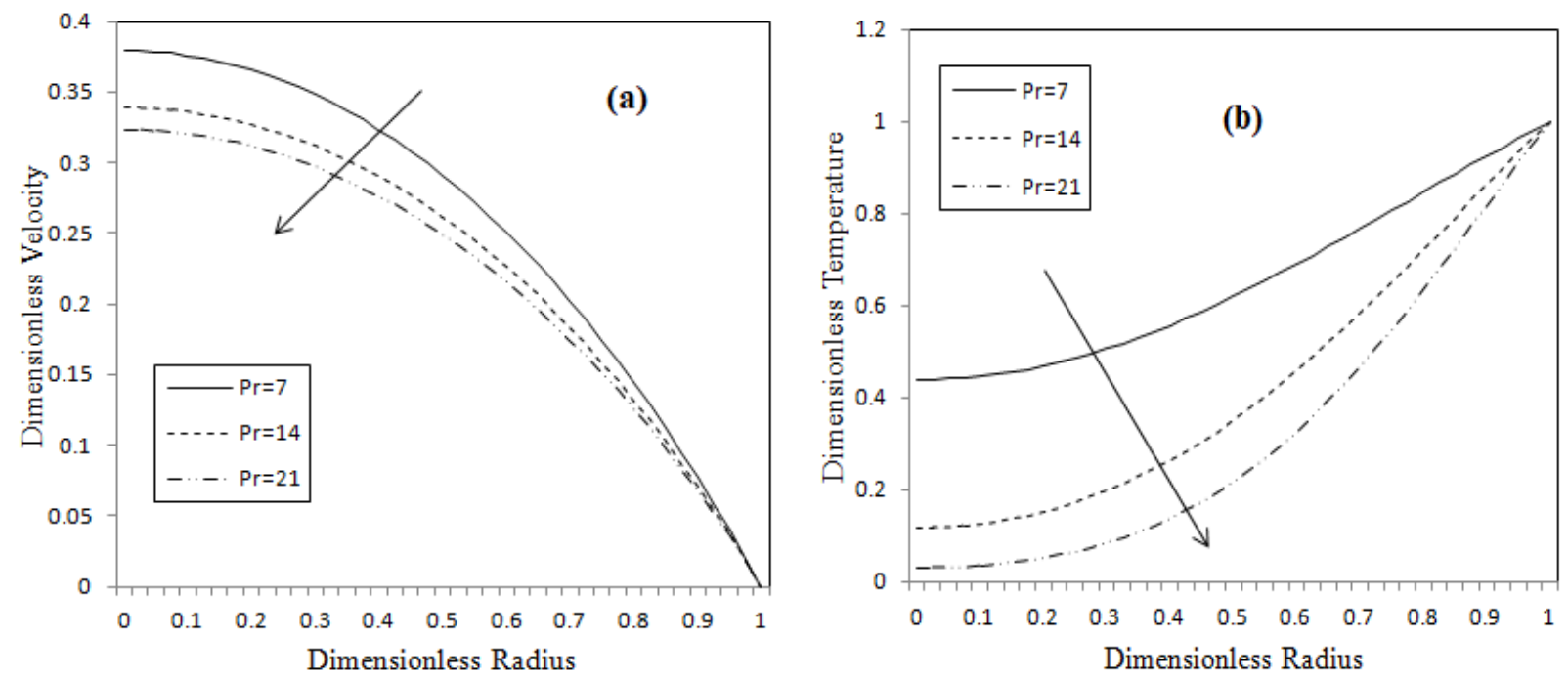

Figure 8: Effect of Prandtl number (Pr) on (a) velocity and (b) temperature profile for following data $B_{1}=1.41, \delta=0.1, z=0.98, t=1.2$

Similarly, the effect of different Reynolds number $(R e)$ on blood flow velocity and temperature profile is elaborated in Figure 9 (a)-(b). It is noteworthy that very low Reynolds numbers are considered (laminar flow), and the regime is, therefore, a viscous-dominated one. The relation represents that on increasing the value of $R e$ from 1 to 10 , a significant amount of reduction in velocity has been seen. In the same manner, as we increased the magnitude of $R e$, the magnitude of temperature is also decreased. Although inertial force is increased with Reynolds number (based on the vessel radius), the overwhelming effect is nevertheless flow deceleration owing to the stenotic obstruction and nanoparticles. 

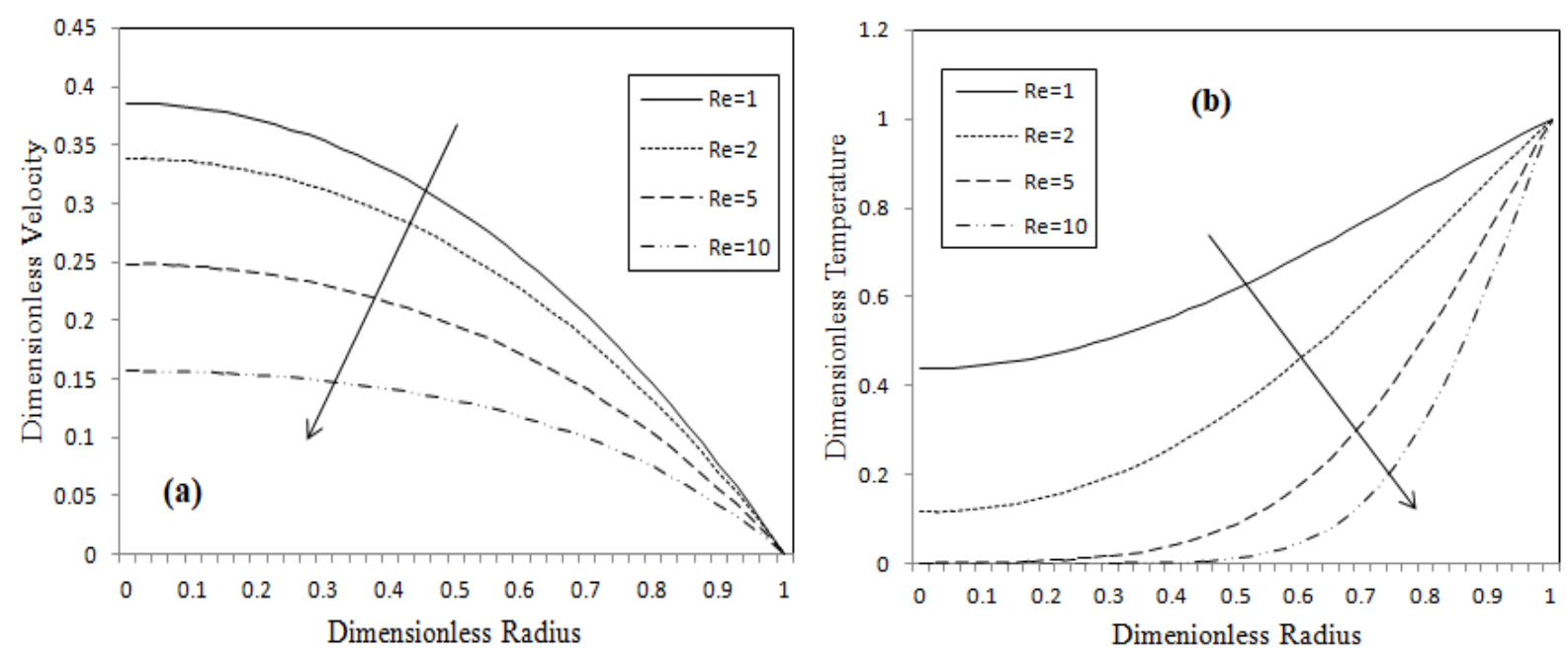

Figure 9: Effect of Reynolds number (Re) on (a) velocity and (b) temperature profile for following data

$$
B_{1}=1.41, \delta=0.1, z=0.98, t=1.2
$$

Figures 10- 23 shows the results for wall shear stress, flow rates and pressure gradient quantities for changing the various parameters. As it is known that wall shear stress is the drag force of the flowing blood exerted on the blood artery's endothelial surface. High shear stress causes laminar flow which promotes the alignment in the flow direction while low shear stress causes turbulent flow and endothelial proliferation, shape changes happen. The volumetric flow rate is the summation (integration) of variable velocity with respect to the radial direction. Hence, the flow rate has the same trend to the axial velocity. Further, pressure gradient shows the variations in pressure value with respect to the time. Nanoscale effects modify the pressure distribution in diseased arterial vessel. This has important implications for controlling the atherosclerosis and other diseases related with stenosis. Figures 10-11 display the time series evolution in wall shear stress (WSS) by varying the nanoparticle concentrations. These figures permit a comparison between the silver nanofluid $\left(\phi_{1}\right)$ and the hybrid nanofluid profile $\left(\phi_{1}+\phi_{2}\right)$. Both the figures show that wall shear stress initially decreases until a critical time and after that increases for the remainder of the time with increasing the volume concentrations for any of the nanoparticles and sustains lower magnitudes for the first part of the cycle at concentration 0.05. However, comparing both figures, it is apparent that wall shear stress is minimized for the case of hybrid nanoparticles $\phi_{1}=0.05, \phi_{2}=0.05$, compared to the case of uni-nanoparticles $\phi_{1}=0.05$. 


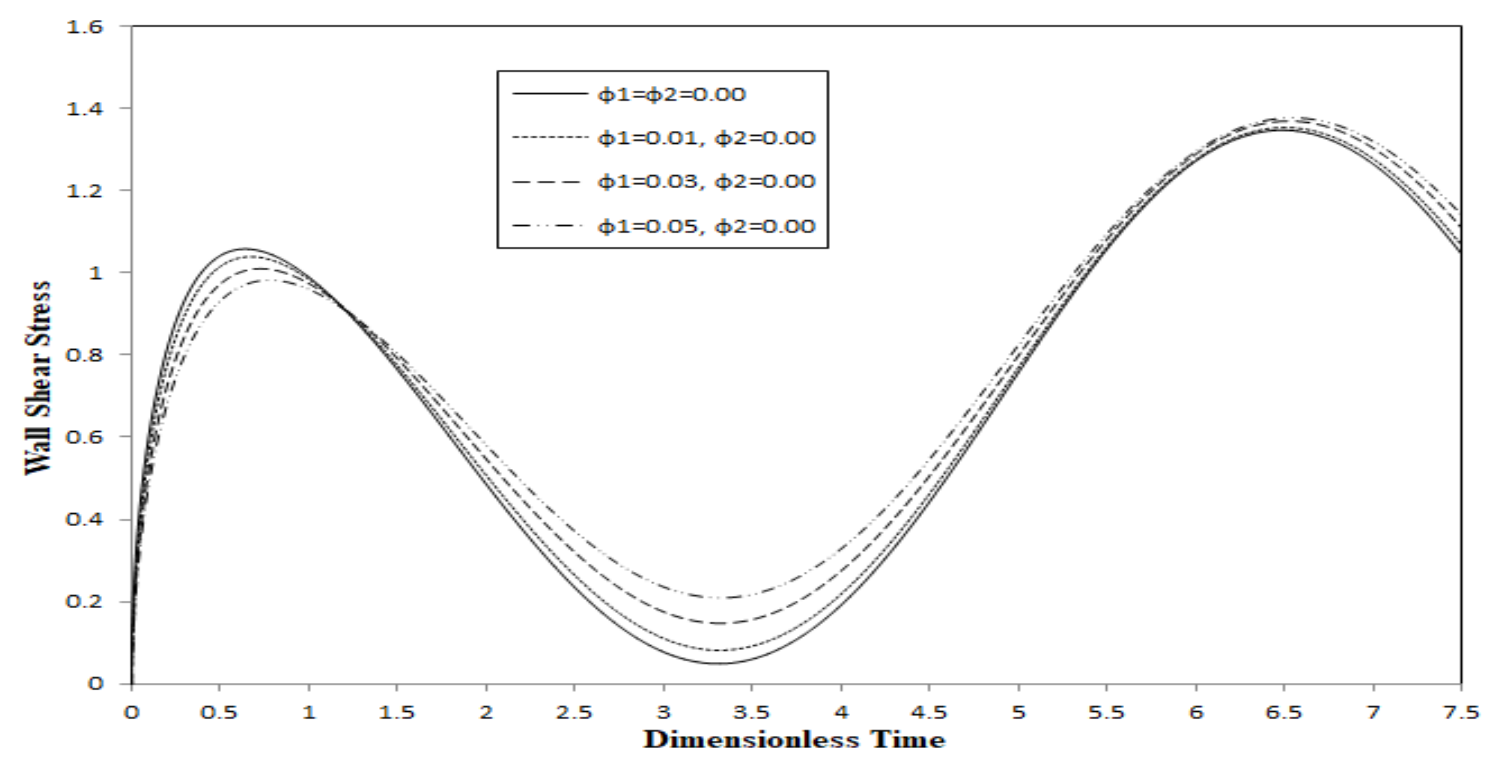

Figure 10: Wall shear stress for various nanoparticle concentration of Ag nanofluid for $B_{1}=1.41, \delta=0.1, z=0.98$

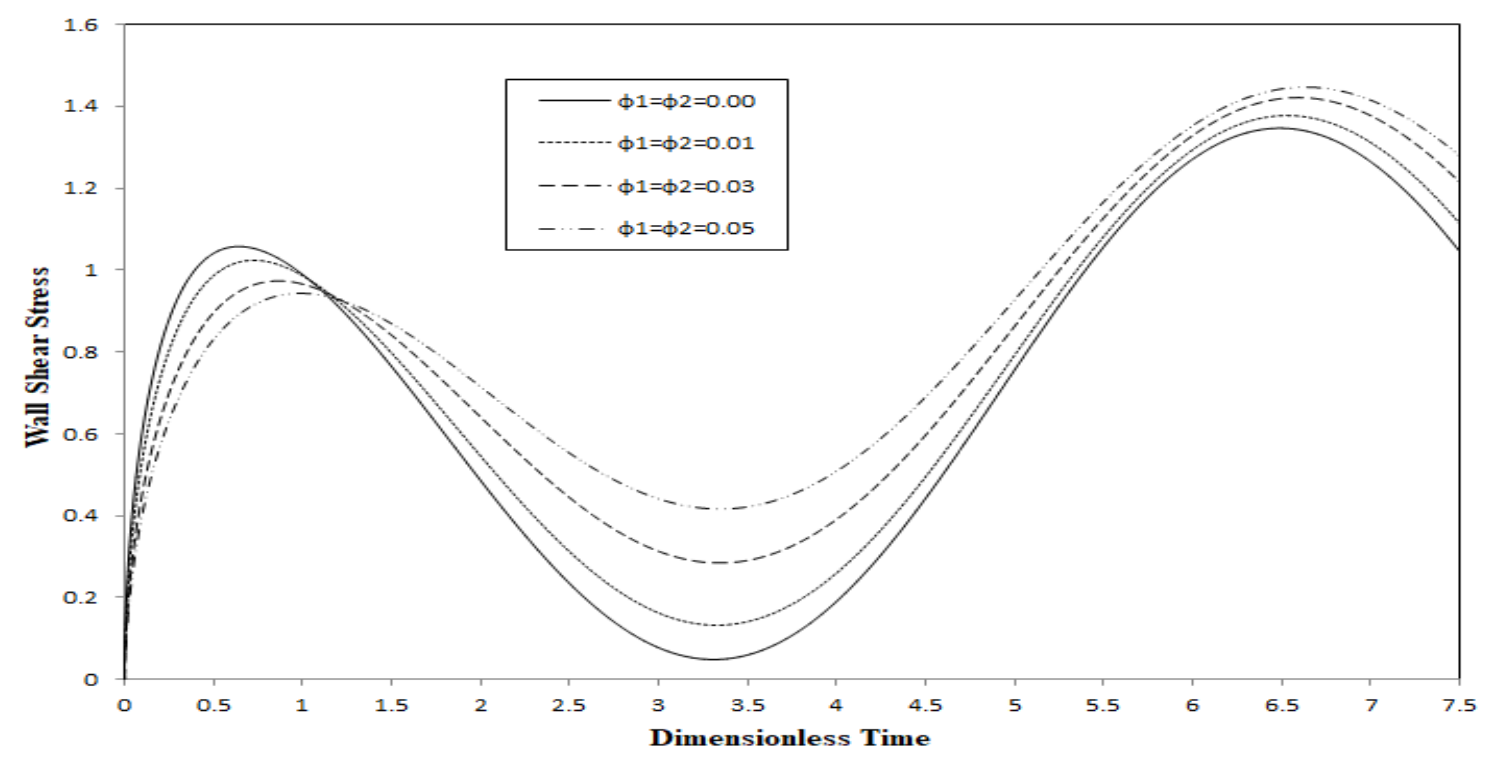

Figure 11: Wall shear stress for various hybrid nanoparticle concentration of $(\mathrm{Ag}+\mathrm{Au})$ nanofluid for

$$
B_{1}=1.41, \delta=0.1, z=0.98
$$

Similarly, Figures (12)-(13) exhibits the impact of various nanoparticle concentrations on the volumetric flow rate. This quantity is also witnessed to be significantly decreasing than increasing function for both silver nanoparticles $\left(\phi_{1}\right)$ and hybrid nanoparticles $\left(\phi_{1}+\phi_{2}\right)$. Following the same trend as wall shear stress, a lower volumetric flow rate is computed; however, for hybrid nanofluid compared with silver nanofluid. 


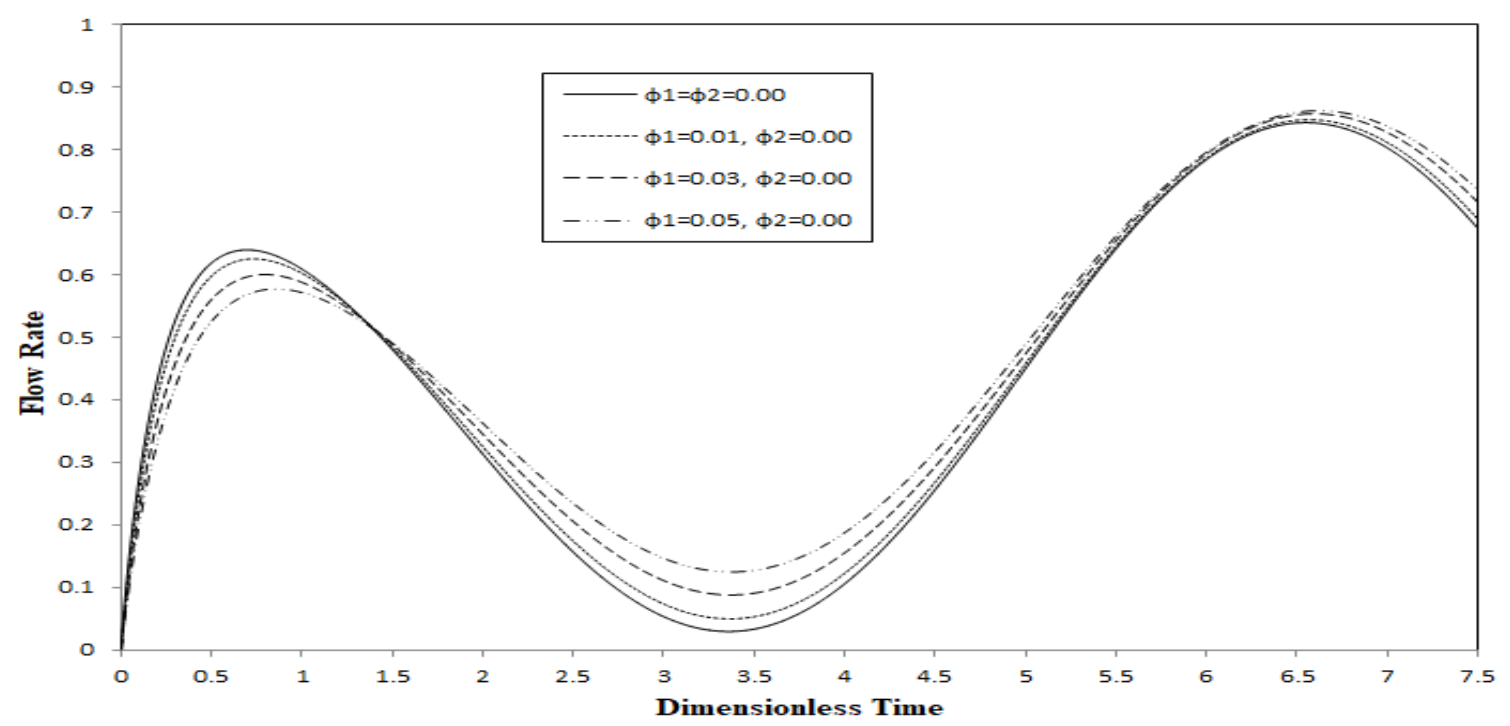

Figure 12: Flow rate for various nanoparticle concentration of $\mathrm{Ag}$ nanofluid for $B_{1}=1.41, \delta=0.1, z=0.98$

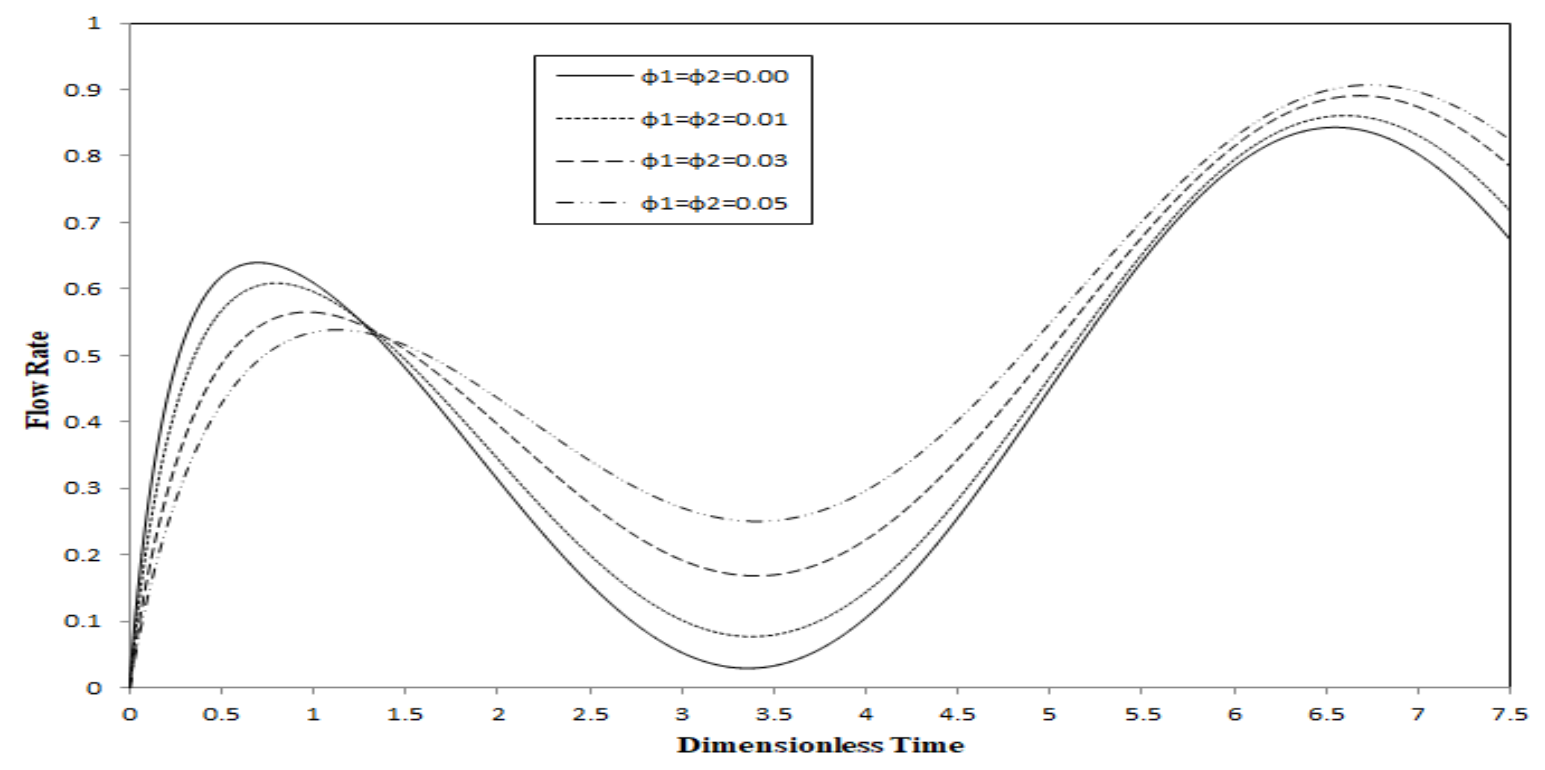

Figure 13: Flow rate for various hybrid nanoparticle concentration of $(\mathrm{Ag}+\mathrm{Au})$ nanofluid for

$$
B_{1}=1.41, \delta=0.1, z=0.98
$$

Figures (14)-(15) shows the changes in the wall shear stress and volumetric flow rate profiles for different combinations of hybrid nanoparticles. The patterns reveal that on increasing the nanoparticle concentration, both profiles first exhibit a reduction in magnitudes up to a particular time and subsequently begin to increase for the remaining duration in time. The variations in the wall shear stress and volumetric flow rate for different values of Grashof number are displayed in 
Figures (16)-(17). From these figures, it is evident that both wall shear stress and volumetric flow rate are enhanced by increasing the magnitude of $G r$, i.e., higher thermal buoyancy force elevates wall shear stress (flow acceleration). It results in more excellent flow rates, which shows a similar increasing pattern to that of the earlier computed velocity profile.

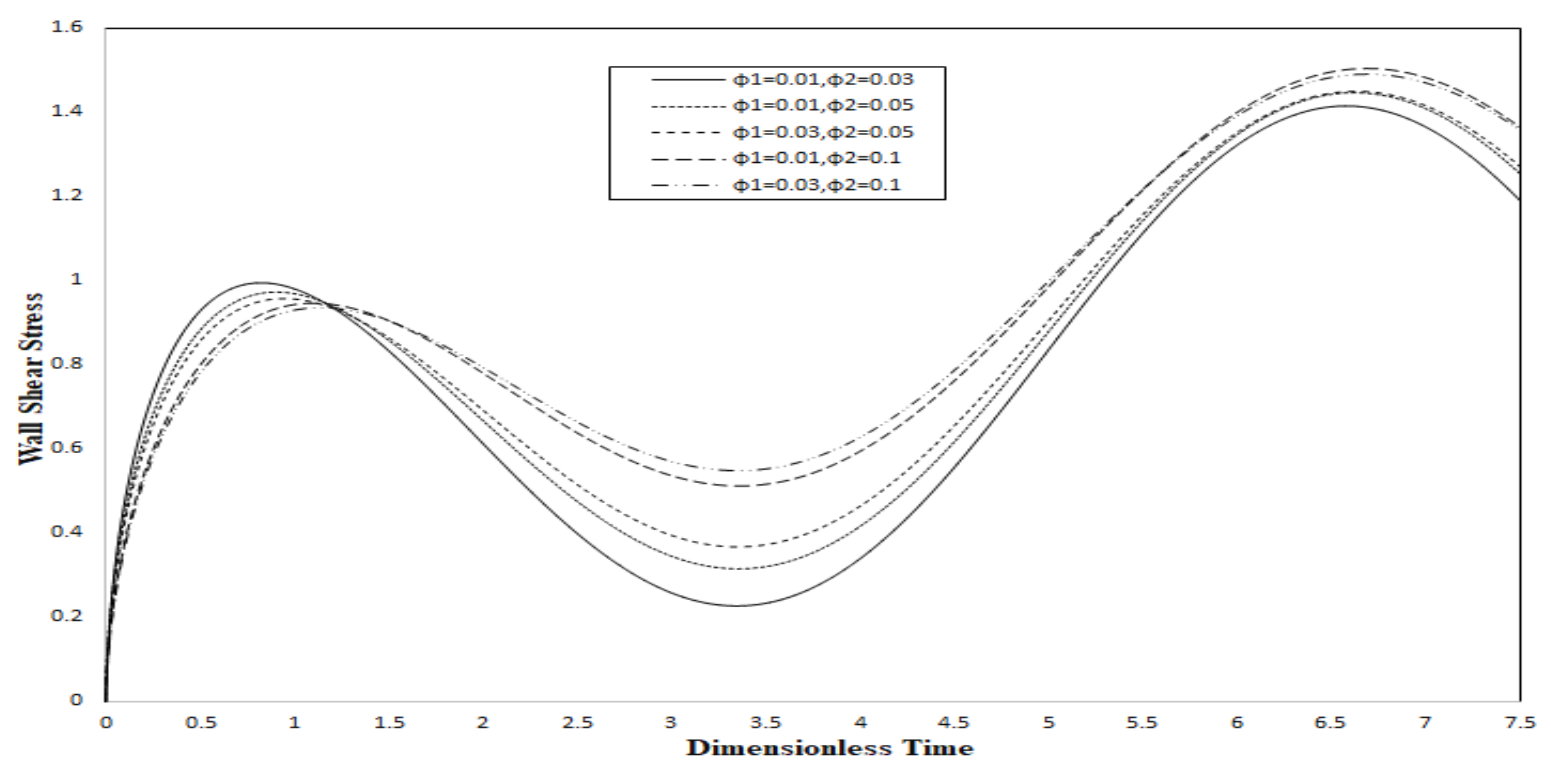

Figure 14: Wall shear stress of different combination of hybrid nanoparticles for $B_{1}=1.41, \delta=0.1, z=0.98$

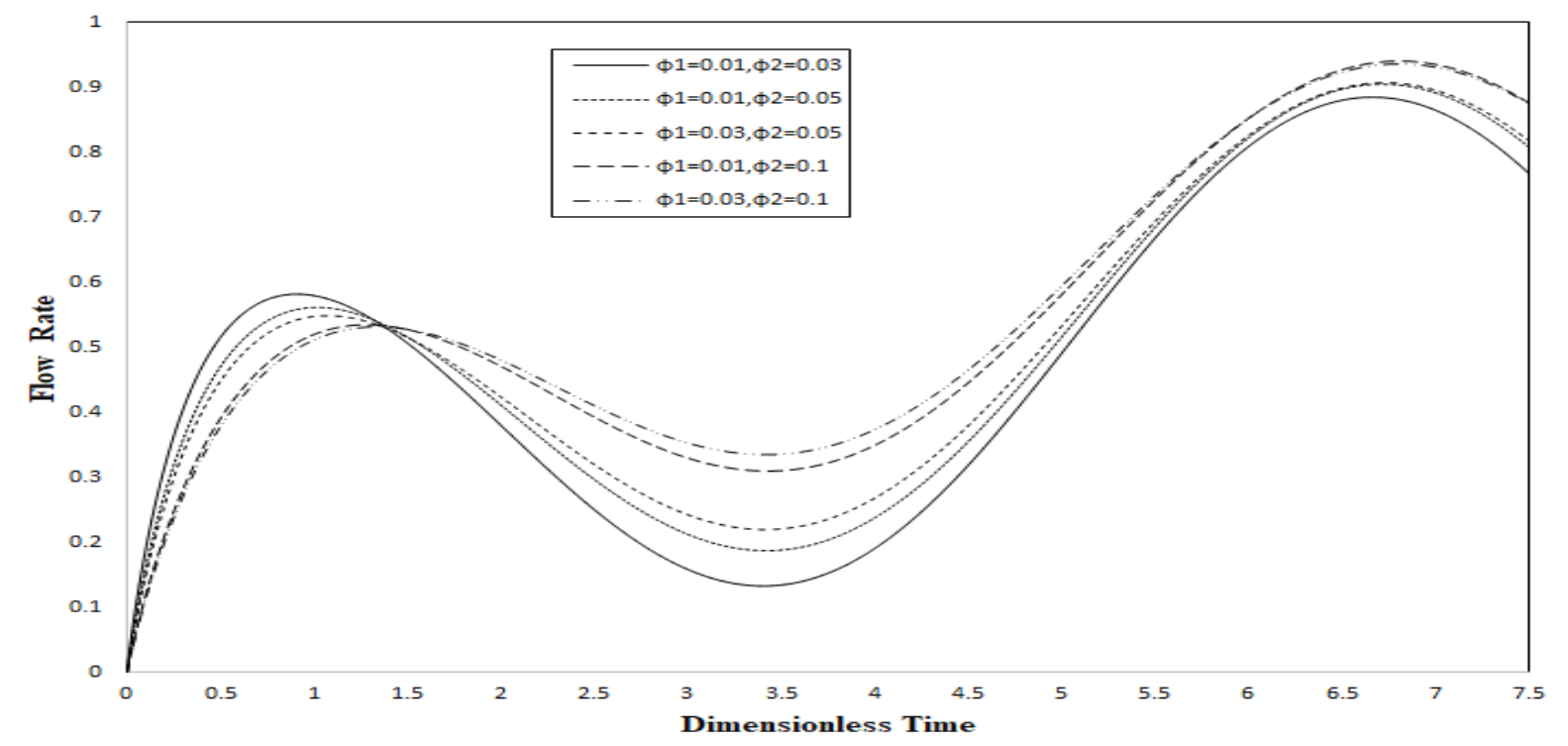

Figure 15: Flow rate of different combination of hybrid nanoparticles for $B_{1}=1.41, \delta=0.1, z=0.98$ 


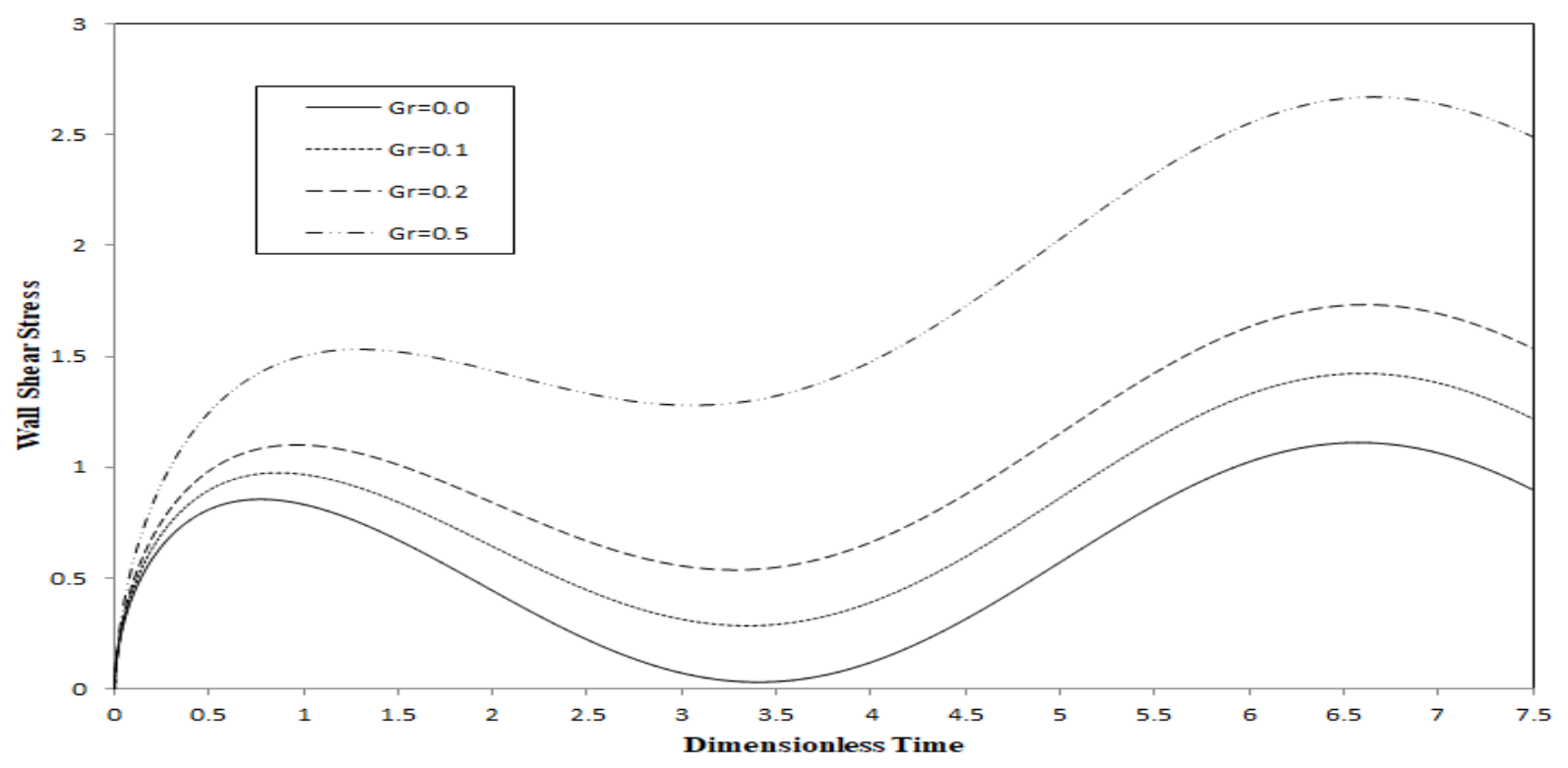

Figure 16: Wall shear stress for various Grashof number for $B_{1}=1.41, \delta=0.1, z=0.98$

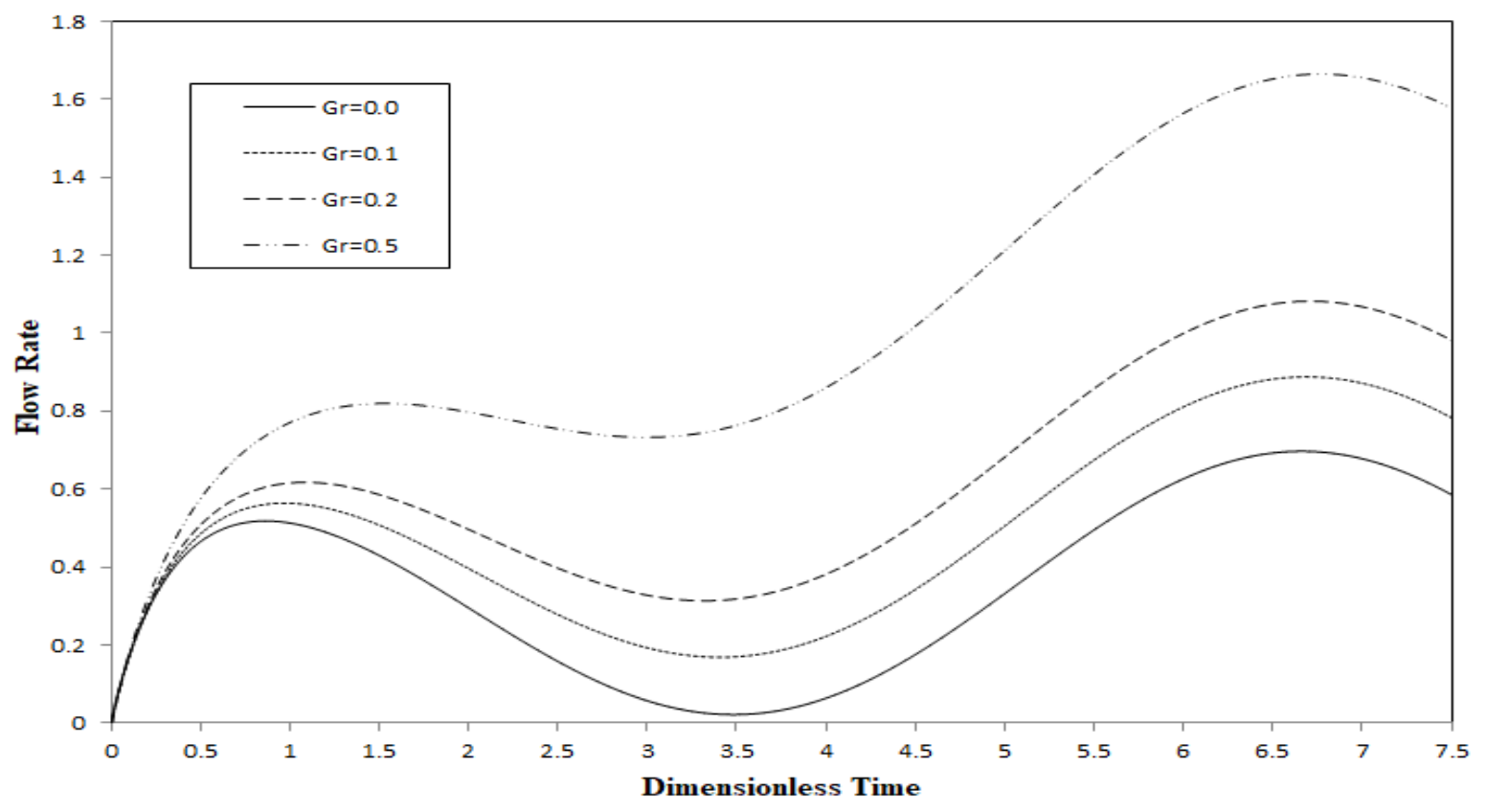

Figure 17: Flow rate for various Grashof number for $B_{1}=1.41, \delta=0.1, z=0.98$ 


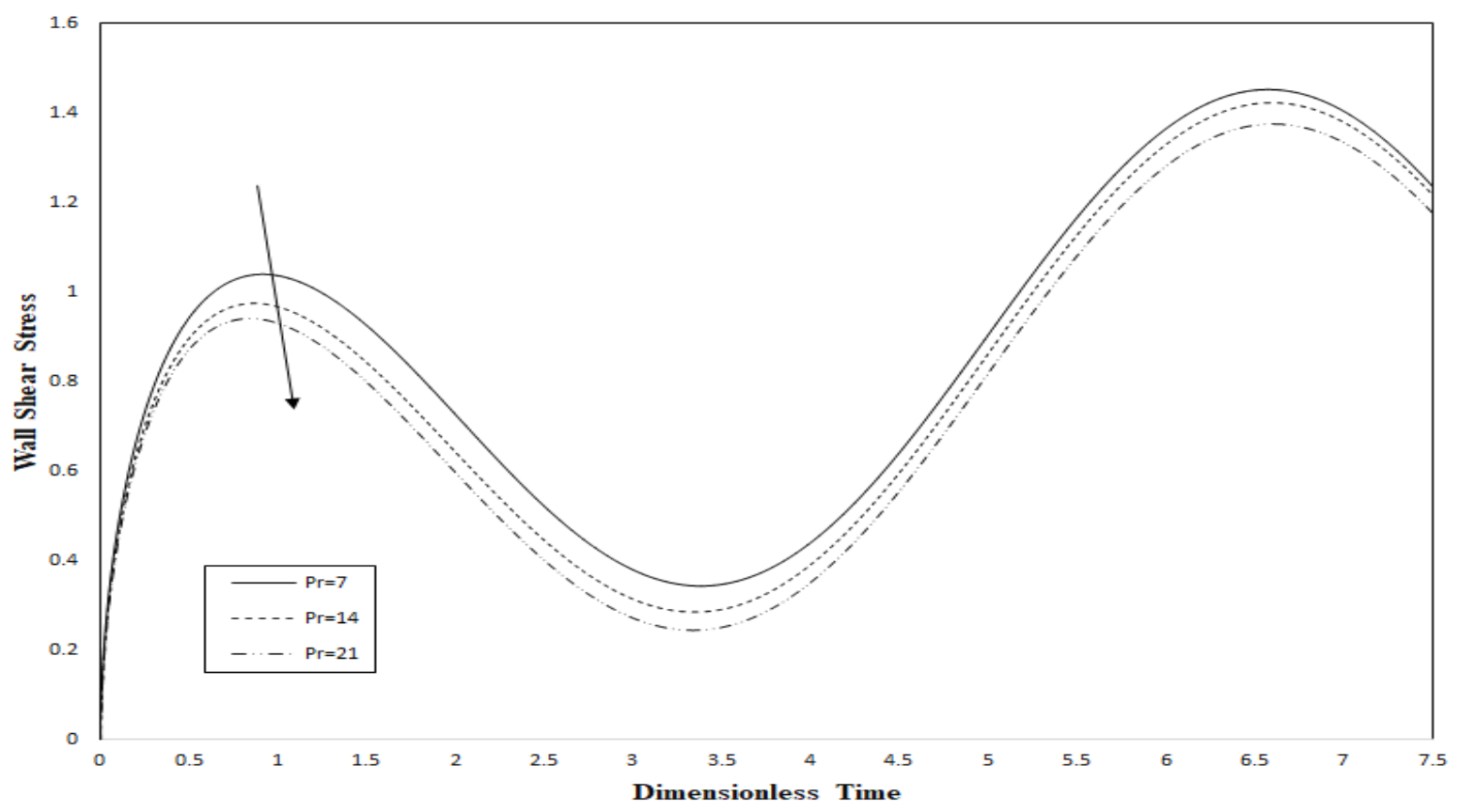

Figure 18: Wall shear stress for various Prandtl number for $B_{1}=1.41, \delta=0.1, z=0.98$

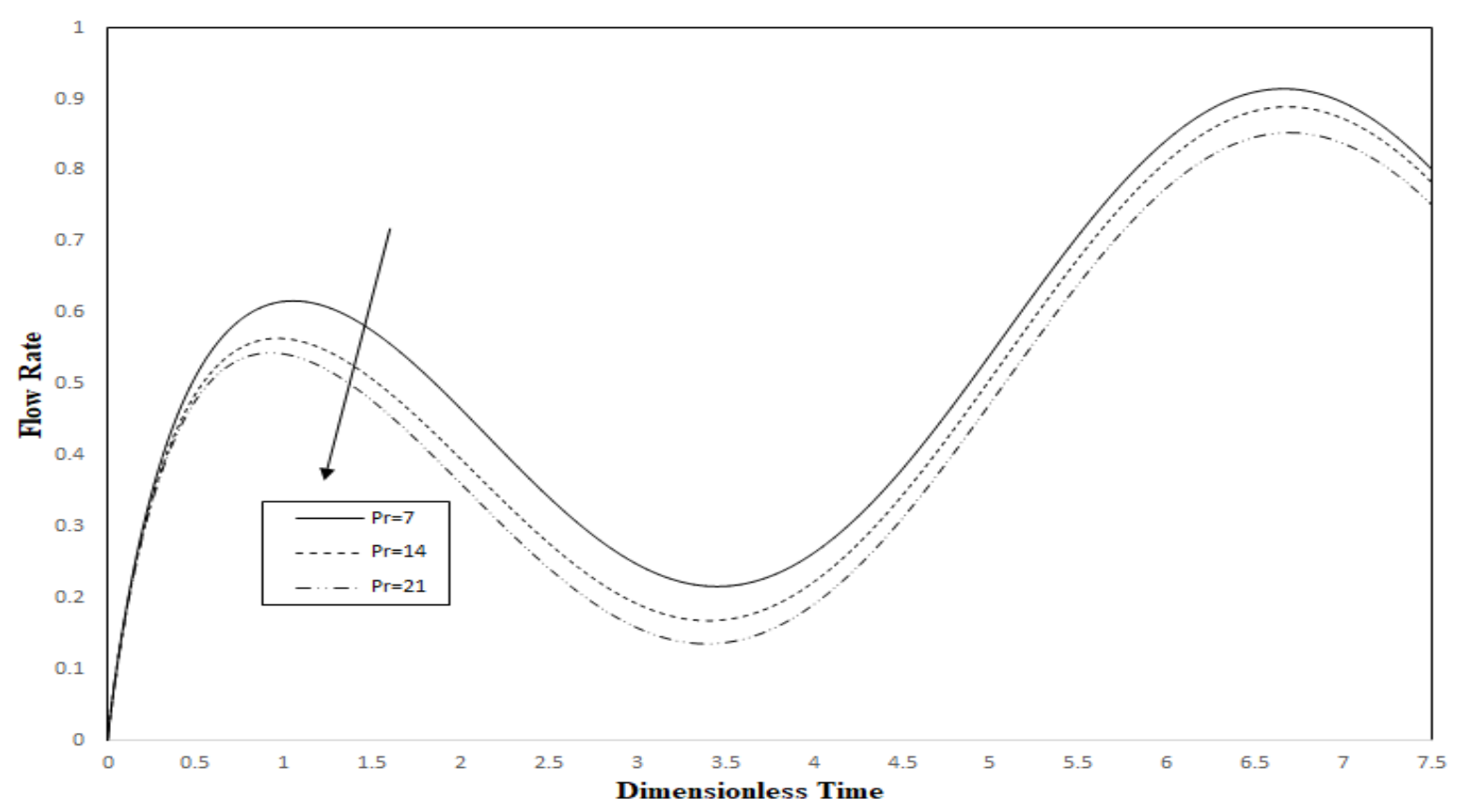

Figure 19: Flow rate for various Prandtl number for $B_{1}=1.41, \delta=0.1, z=0.98$

Figures (18)-(19) depict the transient wall shear stress and volumetric flow rate distributions respectively for increment in Prandtl number $(P r)$. These figures reveal that both the wall shear 
stress and flow rate are depressed noticeably with larger values of Prandtl number. Similar behaviour is also observed by enhancing the value of Reynolds number $(R e)$, which is displayed in Figures (20)-(21). On increasing the value of Reynolds number, the values of wall shear stress and flow rate have lower impacts for higher values of $R e$, following the same trend as axial velocity. The stenotic obstruction and nanoparticle presence counteract the inertial force effect, which manifests in flow deceleration and flow rate drop.

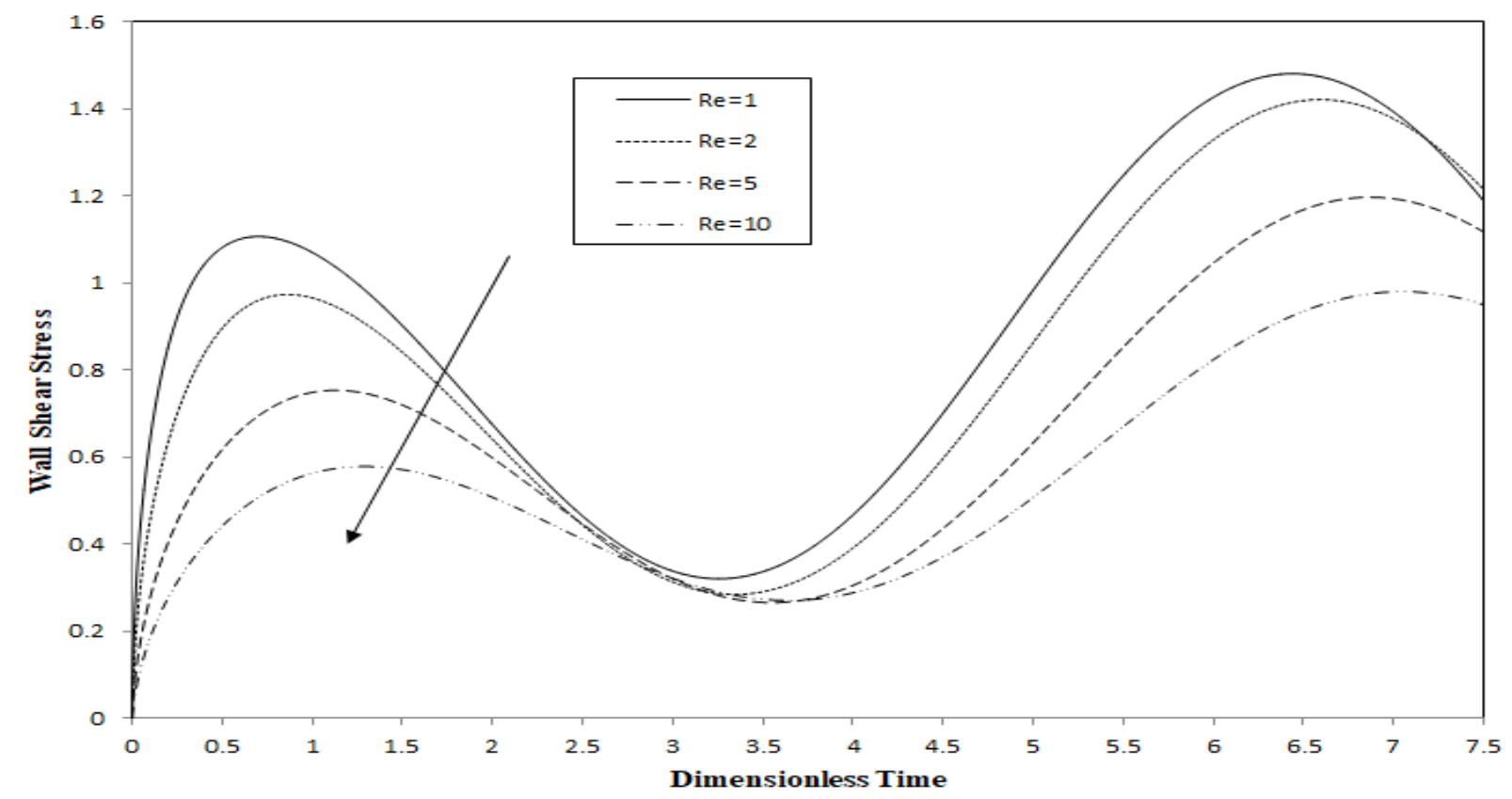

Figure 20: Wall shear stress for various Reynolds number for $B_{1}=1.41, \delta=0.1, z=0.98$

The effect of the $B_{1}$ parameter on the pressure gradient is shown in Figure 22. The graph discloses that on increasing the value of $B 1$, the highest value computed in the pressure gradient cycle (amplitude) is also being elevated. Additionally, Figure 23 represents the effect of the $\mathrm{C}_{1}$ parameter on a pressure gradient profile. It can be seen that on increasing the $\mathrm{C}_{1}$ value, the length of the pressure gradient cycle is reduced, or alternatively, for higher $\mathrm{C}_{1}$, the cycle frequency is increased.

Figure 24 displayed the time profiles of resistance to flow at the stenosis throat for considered set of parameters values. The impedance profile shows opposite trend to volumetric flow rates as from mathematical equation given in Eq. (28), it is clear that both the profiles are inversely related to each other. The solid line ( $G r=0.1$ ) shows the impedance for the default set of parameters given 
in Table 3. The results reveal that the magnitude of resistance impedance decreases by increasing the value of parameters ( $G r, \eta_{0}$ and hybrid nanoparticle concentration) while it increases on increasing the Revalue or for silver nanofluid.

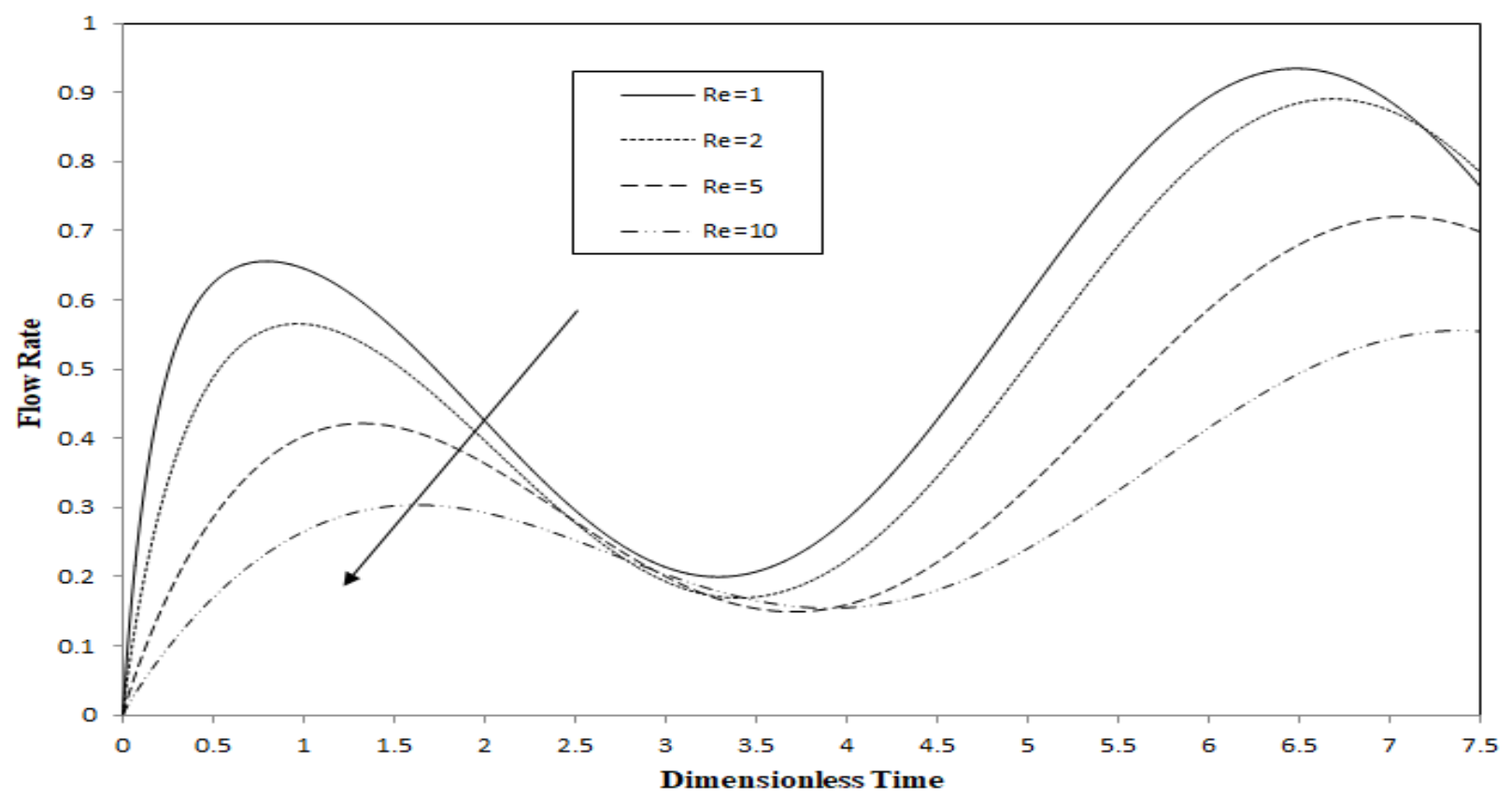

Figure 21: Flow rate for various Reynolds number for $B_{1}=1.41, \delta=0.1, z=0.98$

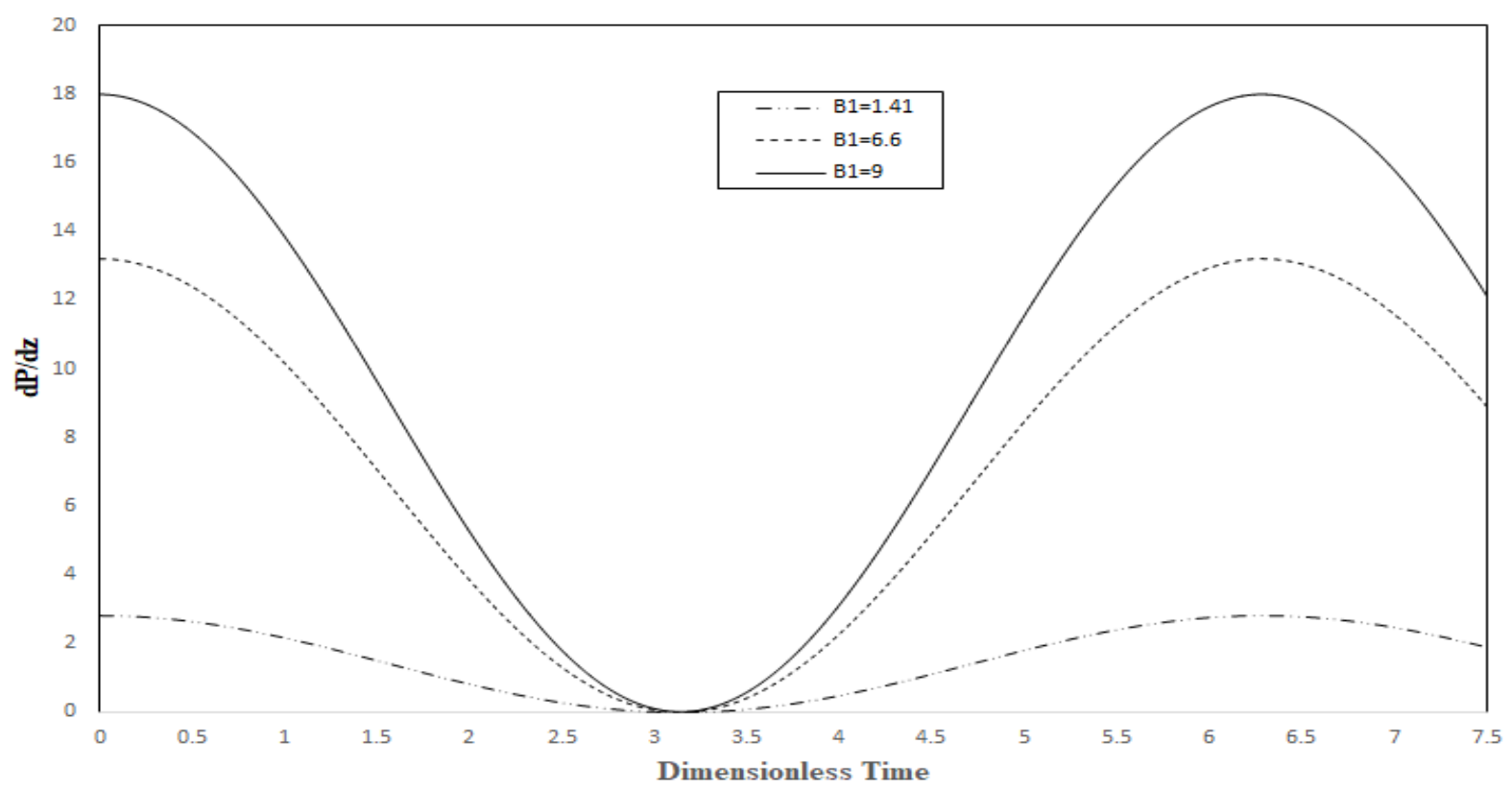

Figure 22: Pressure gradient for various $B 1$ parameter for $c_{1}=1.41, \delta=0.1, z=0.98$ 


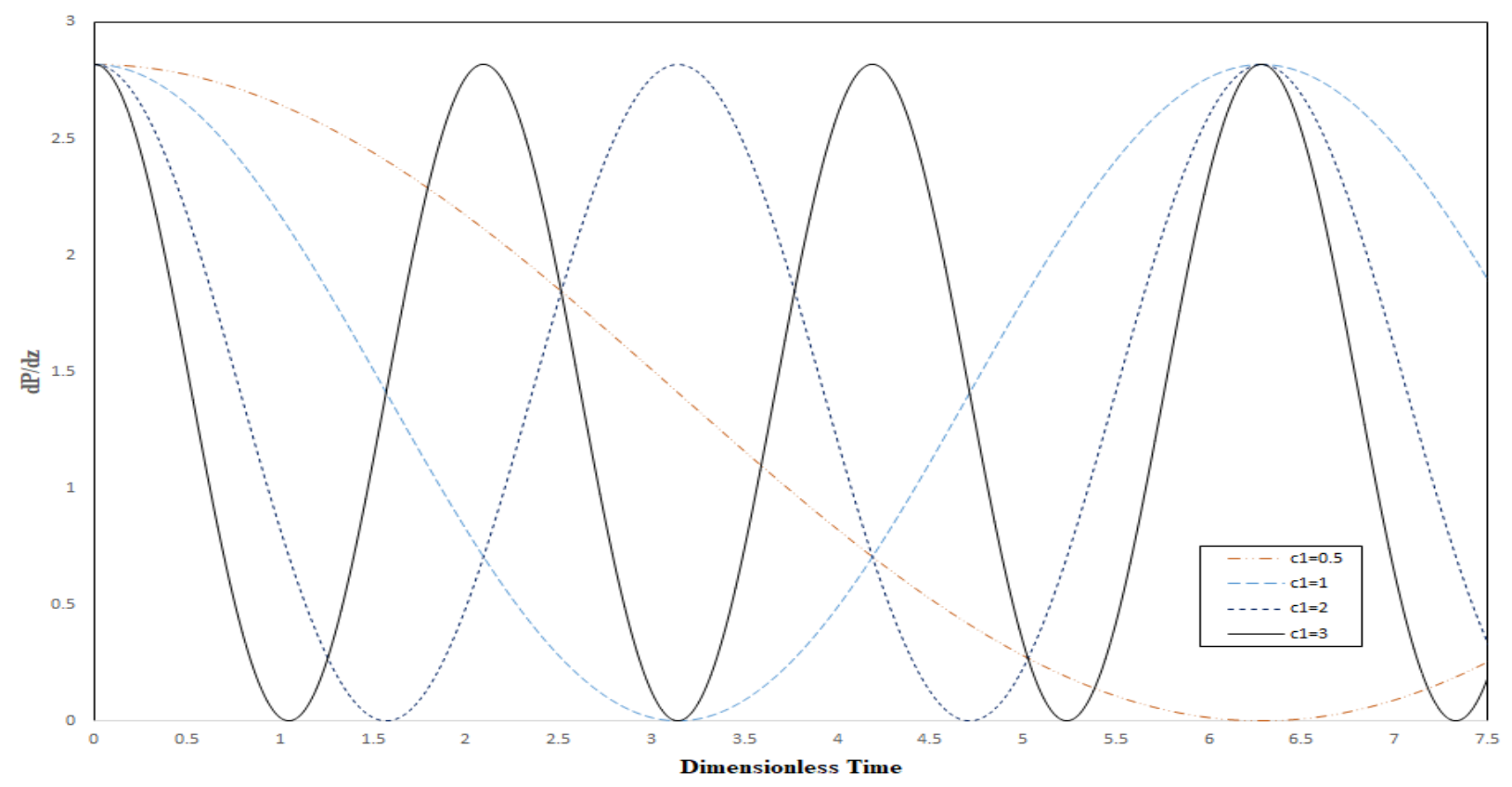

Figure 23: Pressure gradient for various $c_{1}$ parameter for $B_{1}=1.41, \delta=0.1, z=0.98$

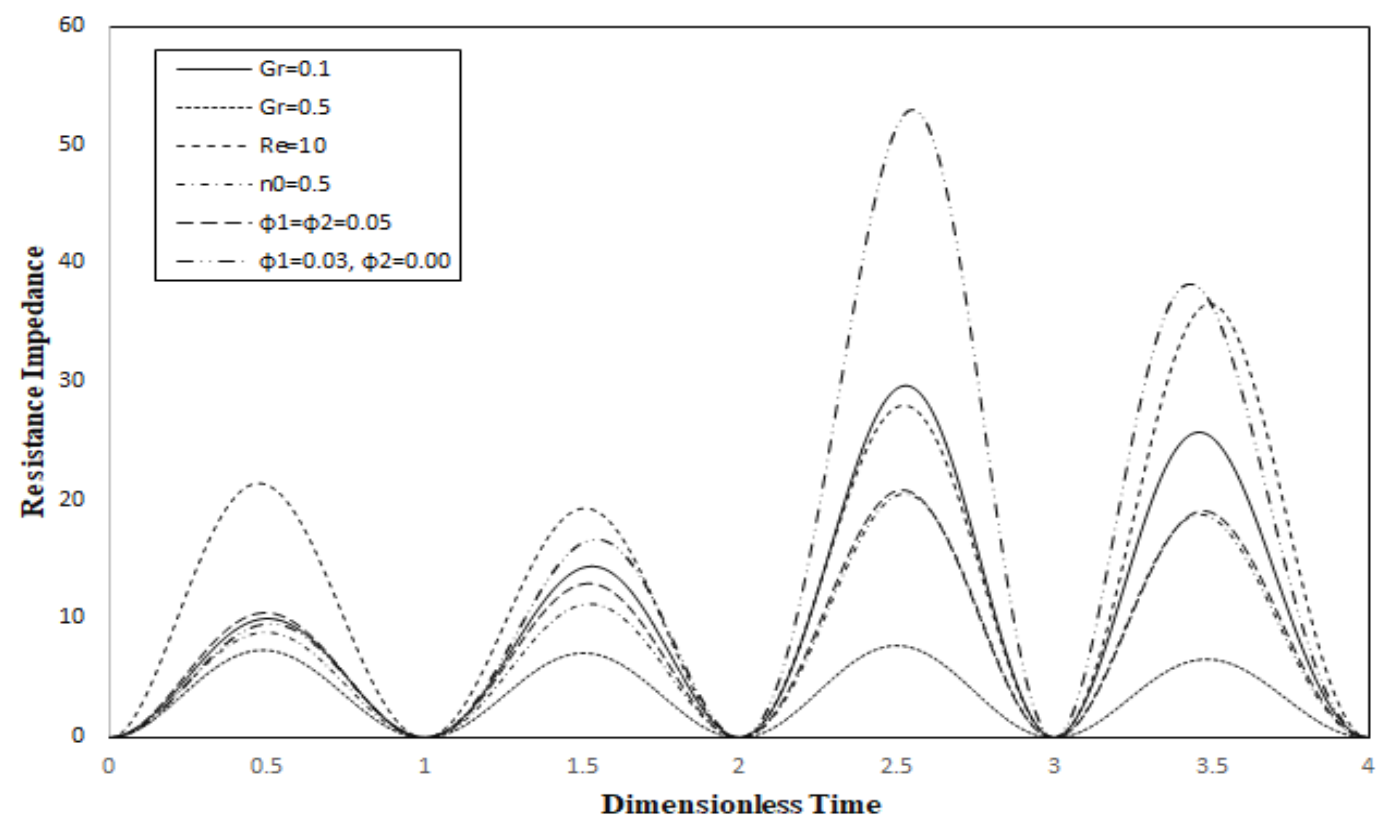

Figure 24: Resistance impedance profile for different set of changed parameters with other default parameter values 

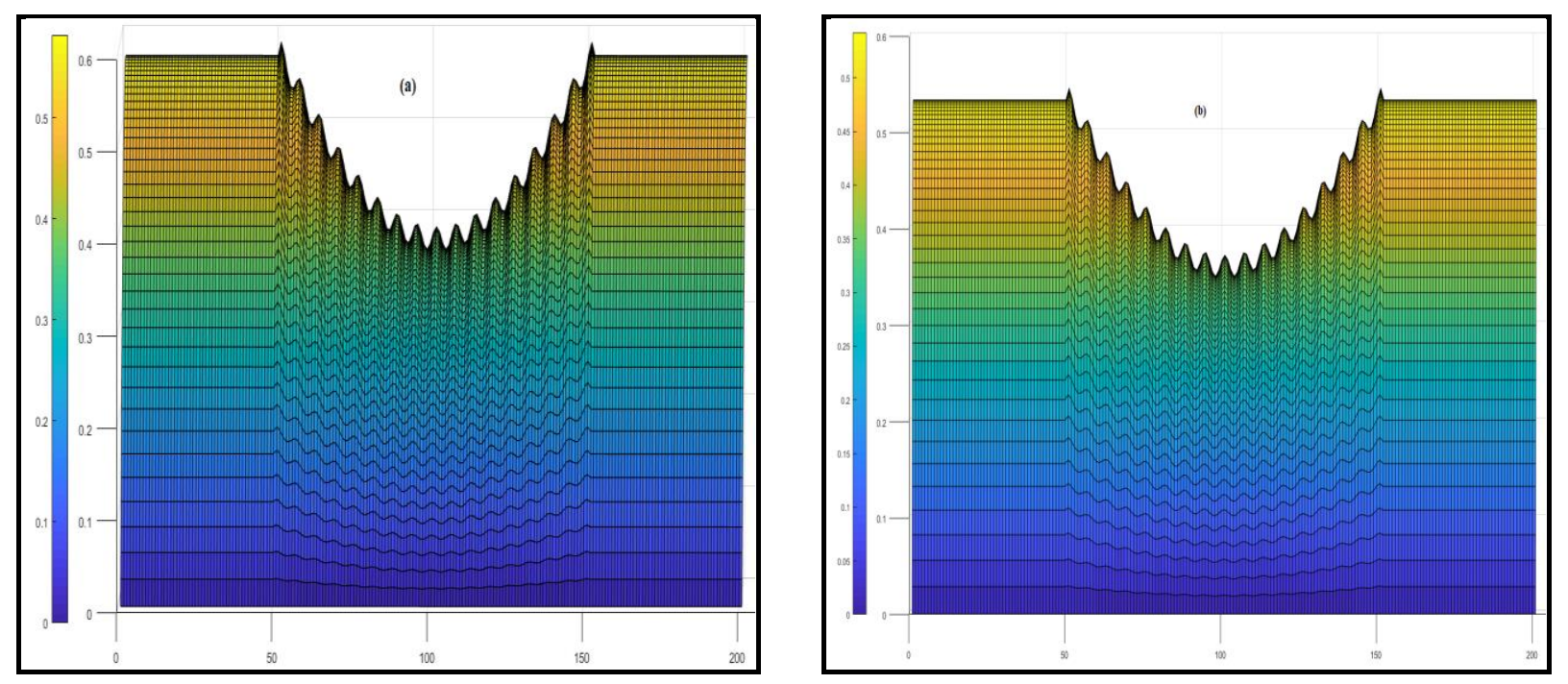

Figure 25: Blood flow pattern along the axial direction with $B_{1}=1.41, \delta=0.1, z=0.98$
(a) For blood $\phi_{1}=0.00, \phi_{2}=0.00$
(b) For silver nano-fluid $\phi_{1}=0.03, \phi_{2}=0.00$

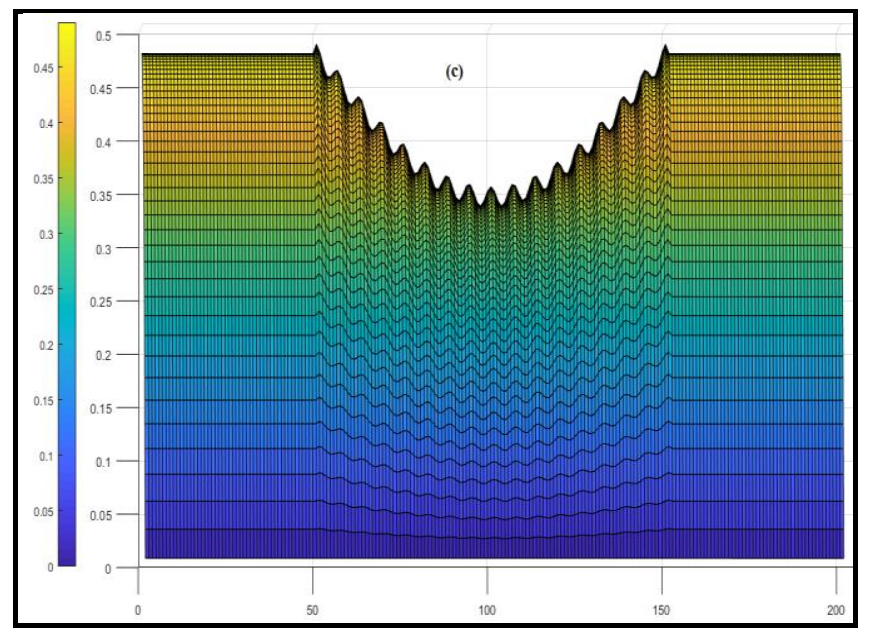

(c) For hybrid nano-fluid $\phi_{1}=0.03, \phi_{2}=0.03$

To analyze the path lines of blood particles along the axial direction, the blood flow patterns are plotted in Figure 25 with different panels (a)-(g). Panel (a) shows the particle pattern for only the case when nanoparticles are not included with the default values of other parameters (i.e., purely viscous fluid model). To calculate the difference in changing the parameters, all the remaining panels, i.e., (b)-(g) are compared with panel (a). Panel (b) displays the pattern for the unitary nanofluid case, i.e., for silver nanoparticle inclusion with the other defaulted parameters value. Similarly, Panel (c) presents the changes for the incorporation of hybrid nanoparticles. From all 
three figures, it is clear that hybrid nanoparticles decrease the velocity and achieve improved hemodynamic control. Panel (d) and (e) visualize the blood flow patterns for changing the value of $\eta_{0}$ from 0.2 to 0.5 and increasing the value of $G r$ from 0.1 to 0.5 , respectively. Compared to the panel (a), it is abundantly clear that blood velocity increases for both cases. Likewise, for $\operatorname{Pr}=21$ and $R e=5$, Panel (f) and (g) indicates that velocity is again a decreasing function of both Prandtl number and Reynolds number in the stenotic regime.

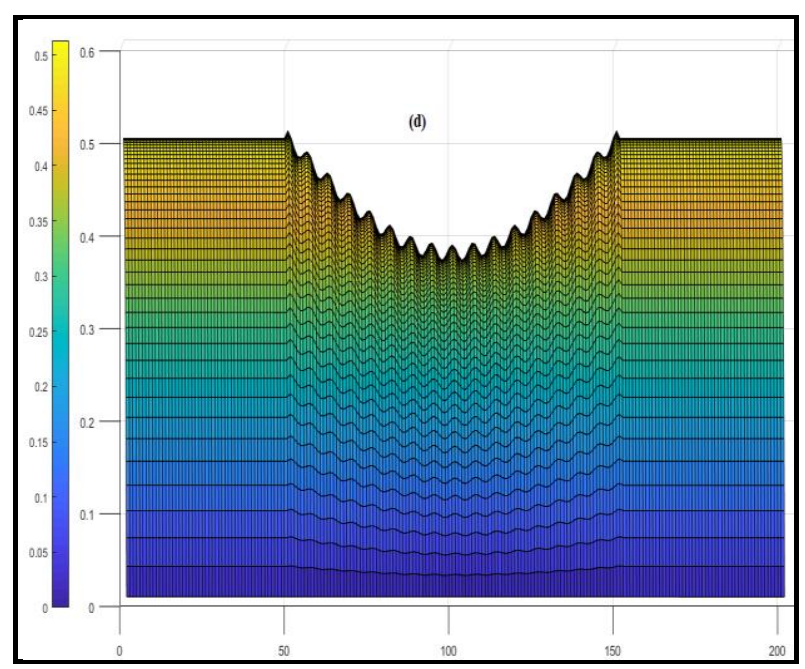

(d) On increasing $\eta_{0}, \eta_{0}=0.5$

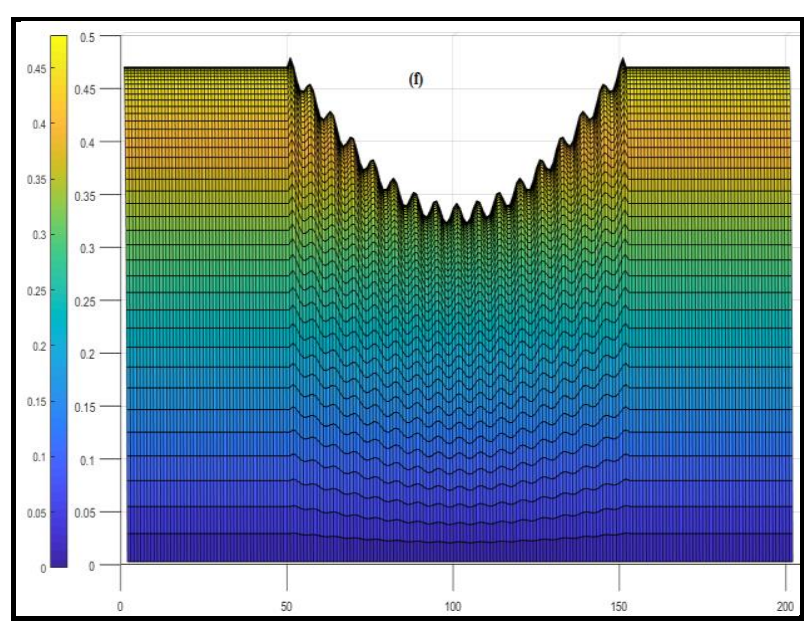

(f) On increasing $\operatorname{Pr}, \operatorname{Pr}=21$

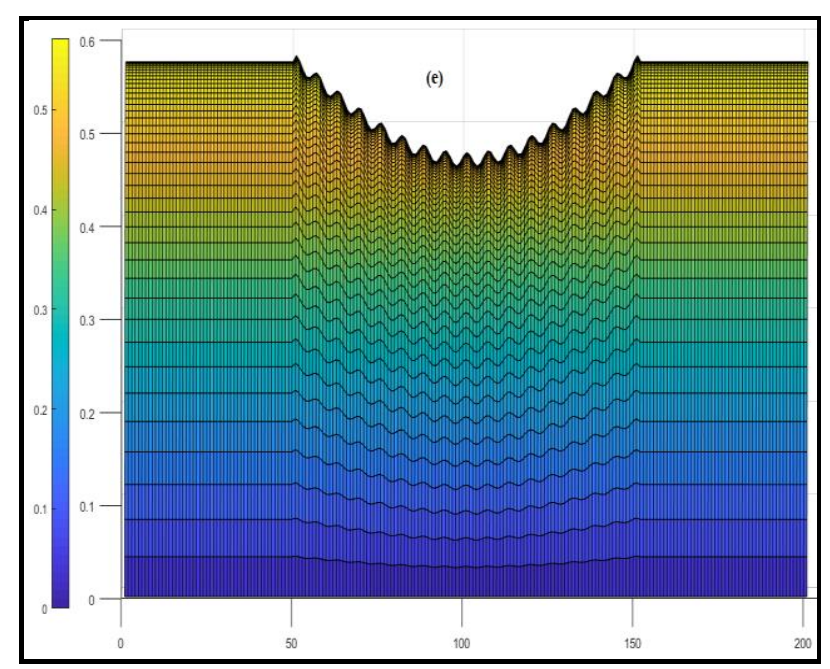

(e) On increasing $G r, G r=0.5$

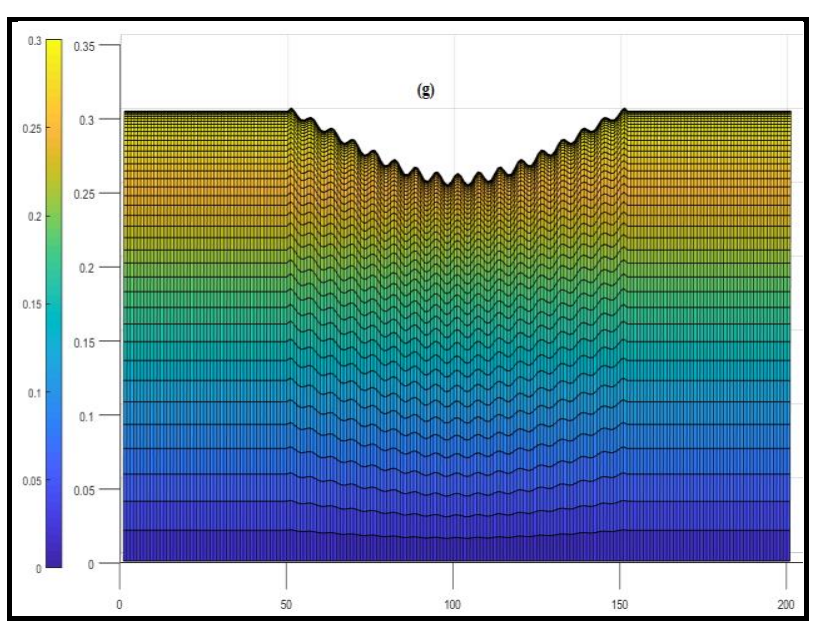

(g) On increasing $\mathrm{Re}, \mathrm{Re}=5$

Tables 4 and 5 demonstrate the variation of wall shear stress and volumetric flow rate for various parameters (viscosity constant $\left(\eta_{0}\right)$ and nanoparticle concentration $\left.(\phi)\right)$ at particular time $t=1.00$. In these tables, the comparison between silver nanofluid and hybrid nanofluid is also presented. The 
data shows that for a fixed nanoparticle volume fraction, the increment in the $\eta_{0}$ increases the both wall shear stress and flow rate profile while for a fixed $\eta_{0}$, both the profiles decrease on increasing the concentration. We also compared the results for silver nanofluid and hybrid nanofluid and it shows that hybrid nanofluid provides more remarkable changes comparing to uni nanofluid for variation of both the parameters.

Table 4: Wall shear stress for various parameters at time $t=1.00$

\begin{tabular}{|c|c|c|c|}
\hline$\eta_{0}$ Variation & $\phi$ Variation & Silver nanofluid & $\begin{array}{l}\text { Hybrid nanofluid } \\
\text { (Ag-Au/Blood) }\end{array}$ \\
\hline \multirow{4}{*}{0.00} & 0.00 & 0.903195798 & 0.903195798 \\
\hline & 0.01 & 0.896956236 & 0.896167283 \\
\hline & 0.03 & 0.883177481 & 0.872505312 \\
\hline & 0.05 & 0.868367924 & 0.846181968 \\
\hline \multirow{4}{*}{0.1} & 0.00 & 0.943605492 & 0.943605492 \\
\hline & 0.01 & 0.937951475 & 0.937760337 \\
\hline & 0.03 & 0.925241631 & 0.915938276 \\
\hline & 0.05 & 0.911393699 & 0.89111918 \\
\hline \multirow{4}{*}{0.2} & 0.00 & 0.989885718 & 0.989885718 \\
\hline & 0.01 & 0.984941737 & 0.985478323 \\
\hline & 0.03 & 0.973535535 & 0.965932828 \\
\hline & 0.05 & 0.960868313 & 0.942998274 \\
\hline \multirow{4}{*}{0.5} & 0.00 & 1.185176361 & 1.185176361 \\
\hline & 0.01 & 1.183688842 & 1.187818402 \\
\hline & 0.03 & 1.17871628 & 1.179904782 \\
\hline & 0.05 & 1.171969926 & 1.166883174 \\
\hline
\end{tabular}


Table 5: Volumetric flow rate for various parameters at time $t=1.00$

\begin{tabular}{|c|c|c|c|}
\hline$\eta_{0}$ Variation & $\phi$ Variation & Silver nanofluid & $\begin{array}{l}\text { Hybrid nanofluid } \\
\text { (Ag-Au/Blood) }\end{array}$ \\
\hline \multirow{4}{*}{0.00} & 0.00 & 0.575692461 & 0.575692461 \\
\hline & 0.01 & 0.567949559 & 0.561943631 \\
\hline & 0.03 & 0.551998591 & 0.529772975 \\
\hline & 0.05 & 0.536027267 & 0.499660854 \\
\hline \multirow{4}{*}{0.1} & 0.00 & 0.591571508 & 0.591571508 \\
\hline & 0.01 & 0.584044924 & 0.578051426 \\
\hline & 0.03 & 0.568506547 & 0.546235249 \\
\hline & 0.05 & 0.552939906 & 0.516551676 \\
\hline \multirow{4}{*}{0.2} & 0.00 & 0.609078049 & 0.609078049 \\
\hline & 0.01 & 0.601796803 & 0.595816409 \\
\hline & 0.03 & 0.586727765 & 0.564409069 \\
\hline & 0.05 & 0.571622745 & 0.535218921 \\
\hline \multirow{4}{*}{0.5} & 0.00 & 0.675804691 & 0.675804691 \\
\hline & 0.01 & 0.669527795 & 0.663604035 \\
\hline & 0.03 & 0.656392448 & 0.633952678 \\
\hline & 0.05 & 0.643199383 & 0.606857576 \\
\hline
\end{tabular}

Figure 26 (a)-(b) displays the variation of axial velocity and temperature for other nanoparticles suspended within the blood. Several combinations of hybrid nanoparticles $\left(\mathrm{Cu}, \mathrm{TiO}_{2}, \mathrm{SiO}_{2}\right)$ are considered and compared. From both graphs, it is apparent that the hybrid combination of (Ag and $\mathrm{Au})$ achieves superior results (lower velocity and temperature) relative to any other hybrid nanoparticle combination. The Ag-Au hybrid case effectively decelerates the blood flow and elevates blood temperature profiles, which is extremely desirable for nano-drug delivery applications [37]. 

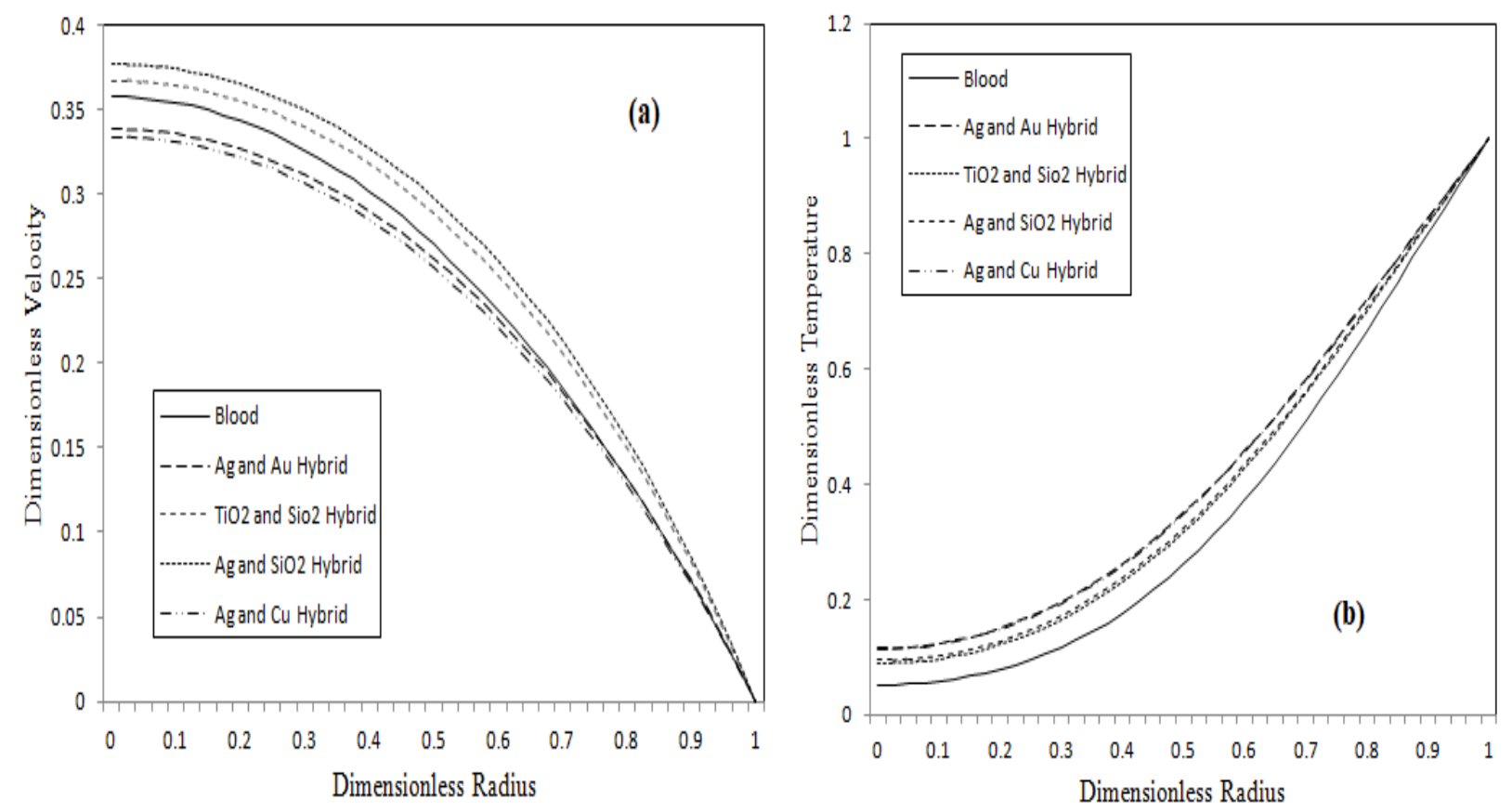

Figure 26: Effect of different types of hybrid nanoparticles on (a) velocity and (b) temperature profile for

$$
B_{1}=1.41, \delta=0.1, z=0.98, t=1.2 .
$$

\section{CONCLUSIONS}

Inspired by nano-drug delivery technologies for arterial disease treatment, this study provides a more profound understanding via computational simulations of hybrid nanoparticles mediated hemodynamic transport (Ag-Au/blood) through an artery with an irregular stenosis. Reynolds' viscosity model is applied in the formulation to represent the temperature dependency of blood. For realistic flow situations, the unsteady component of the pulsatile pressure gradient is also included. Further, quantifying the blood flow characteristics (velocity, temperature, wall shear stress, volumetric flow rate and pressure) at the stenotic region, the non-dimensional, transformed governing conservation equations are solved with the help of an FTCS finite difference method. The key findings of the present numerical computations may be summarized as follows:

- It has been observed that the inclusion of hybrid nanoparticles (Ag-Au/blood) within blood decreases the axial velocity of blood more significantly as compared to unitary nanoparticles (Ag/blood). Also, the temperature profile increases noticeably on varying the concentration of hybrid nanoparticles. 
- Enhancement in thermal buoyancy effect as simulated via Grashof number $(G r)$ and increasing viscosity parameter $\left(\eta_{0}\right)$, both induce considerable axial flow acceleration in the stenotic regime while no impact has been shown for temperature profile.

- Both velocity profile and temperature magnitudes are depressed with rising values of Prandtl number $(P r)$, i.e., lower thermal conductivity, and with increasing Reynolds number $(R e)$, i.e., greater inertial force in the stenotic regime.

- On increasing the value of pressure gradient parameter $\left(B_{l}\right)$, velocity is increased, whereas no tangible modification is computed in temperature. Whereas, on increasing the pressure parameter $\left(c_{1}\right)$, axial velocity is observed to follow the descending pattern.

- The wall shear stress and volumetric flow rate values have substantially greater magnitudes for hybrid nanofluids comparing to unitary nanofluid.

- Enhancement in Grashof number increases the both wall shear stress (WSS) and flow rate (FR) profile. Furthermore, these two profiles (WSS and FR) are depressed with ascending values of Prandtl number (Pr) and Reynolds number (Re).

- With time variation, the pressure gradient is found to be lower in coronary (small) arteries comparatively to large (e.g., femoral) arteries.

- Variation in wall shear stress and flow rate for changing the emerging parameters such as viscosity constant and nanoparticle concentration is shown in Table 3 and 4 . The data also shows the comparison between the silver nanofluid and hybrid nanofluid at the throat of the stenosis.

- The comparison for distinct hybrid nanofluids indicates that (Ag-Au/-nanoparticle doped blood) produces more beneficial results for nano-drug delivery therapies since it achieves improved hemodynamic flow control and higher temperatures in streaming blood.

The present simulations have been confined to electrically non-conducting blood, i.e., they have ignored electro-osmotic (and biomagnetic) characteristics of blood flows [38], which are also of interest to nano-drug delivery. These feature the application of external electrical and magnetic fields to manipulate nanoparticle distribution in the human body (generating electro-kinetic and ferromagnetic dipole effects) and may be explored, in the near future.

This article has been motivated by growing clinical applications of targeted nano-drug delivery systems. The advantage of numerical blood flow simulation is that it provides almost limitless (and 
relatively inexpensive) insights which can aid decision-making processes during the treatment of cardiovascular diseases. Although a conventional method for treating the stenosis is to deploy a stent or catheter inside the artery, however, nowadays targeting the nano-drugs at desired locations is increasingly becoming the new standard. This also triggers the process of clotting formation at the stenosis throat and the effects of such post-treatment processes can also be predicted by computational simulation.

\section{ACKNOWLEDGEMENTS}

The authors are grateful to the Science and Engineering Research Board (SERB), Department of Science and Technology (DST), government of India for undertaking the research work under the research project File Number: ECR/2017/001053 dated 12/03/2018. Prof. Anwar Bég would also like to thank Prof. Niroshini Nirmylan of Biomolecular Sciences, Salford University for some useful discussions on nano-hemodynamics. The authors appreciate the comments of all 3 reviewers which have served to improve the present work.

\section{FUNDING}

This research received no funding.

\section{CONFLICT OF INTEREST STATEMENT:}

The authors have no conflict of interest in the subject matter discussed in the manuscript.

\section{REFERENCES:}

[1] Young, D. F., \& Tsai, F. Y. (1973). Flow characteristics in models of arterial stenoses-I. Steady flow. Journal of Biomechanics, 6(4), 395-410.

[2] Johnston, P. R., \& Kilpatrick, D. (1991). Mathematical modelling of flow through an irregular arterial stenosis. Journal of Biomechanics, 24(11), 1069-1077. 
[3] Chakravarty, S., \& Mandal, P. K. (1994). Mathematical modelling of blood flow through an overlapping arterial stenosis. Mathematical and Computer Modelling, 19(1), 59-70.

[4] Mustapha, N., Mandal, P. K., Johnston, P. R., \& Amin, N. (2010). A numerical simulation of unsteady blood flow through multi-irregular arterial stenoses. Applied Mathematical Modelling, 34(6), 1559-1573.

[5] Abdullah, I., Amin, N., \& Hayat, T. (2011). Magnetohydrodynamic effects on blood flow through an irregular stenosis. International Journal for Numerical Methods in Fluids, 67(11), 1624-1636.

[6] Ellahi, R., Rahman, S. U., Gulzar, M. M., Nadeem, S., Vafai, K. (2014). A mathematical study of non-Newtonian micropolar fluid in arterial blood flow through composite stenosis. Applied Mathematics and Information Sciences, 8(4), 1567.

[7] Zaman, A., Ali, N., \& Bég, O. Anwar (2016). Numerical simulation of unsteady micropolar hemodynamics in a tapered catheterized artery with a combination of stenosis and aneurysm. Medical \& Biological Engineering \& Computing, 54(9), 1423-1436.

[8] Banach, M., Serban, C., Sahebkar, A., Mikhailidis, D. P., Ursoniu, S., Ray, K. K., ... and Garcia-Garcia, H. M., Impact of statin therapy on coronary plaque composition: a systematic review and meta-analysis of virtual histology intravascular ultrasound studies. BMC medicine, vol. 13, no. 1, 229, 2015.

[9] Choi S U S, Eastman J A. Enhancing thermal conductivity of fluids with nanoparticles. ASME Int Mech Eng Congr Exp 1995;66: 99-105

[10] Wagner V, Dullaart A, Bock AK, Zweck A. The emerging nanomedicine landscape, Nat Biotechnol. 2006; 24:10

[11] Tripathi, D., Bég, O. Anwar (2014). A study on peristaltic flow of nanofluids: Application in drug delivery systems. International Journal of Heat and Mass Transfer, 70, 61-70.

[12] Tripathi, J., Vasu, B., Dubey, A., Gorla, R. S. R., Murthy, P. V. S. N., Bég, O. A., \& Saikrishnan, P. (2020). A review on recent advancements in the hemodynamics of nano-drug delivery systems. Nanoscience and Technology: An International Journal, 11(1), 73-98 (2020).

[13] Vasu, B., Dubey, A., Bég, O. Anwar (2019). Finite element analysis of non-Newtonian magneto-hemodynamic flow conveying nanoparticles through a stenosed coronary artery. Heat Transfer, 49 (1) 33-66 (2020). 
[14] Ahmed, A., \& Nadeem, S. (2016). The study of (Cu, TiO2, Al2O3) nanoparticles as antimicrobials of blood flow through diseased arteries. Journal of Molecular Liquids, 216, 615-623.

[15] Dubey, A., Vasu, B., Bég, O. Anwar, Gorla, R. S., \& Kadir, A. (2020). Computational fluid dynamic simulation of two-fluid non-Newtonian nanohemodynamics through a diseased artery with a stenosis and aneurysm. Computer Methods in Biomechanics and Biomedical Engineering, 1-27. DOI: 10.1080/10255842.2020.1729755 (28 pages)

[16] Tariq, H., Khan, A. A., \& Zaman, A. Theoretical Analysis of Peristaltic Viscous Fluid with Inhomogeneous Dust Particles. mechanics, 10, 23.

[17] Zaman, A., \& Khan, A. A. Time dependent non-Newtonian nano-fluid (blood) flow in wshape stenosed channel; with curvature effects. Mathematics and Computers in Simulation, 181, 82-97.

[18] A. Makisima, Possibility of hybrids materials, Ceram. Japan. 39 (2004) 90-91.

[19] Ijaz, S., \& Nadeem, S. (2017). Biomedical theoretical investigation of blood mediated nanoparticles (Ag-Al2O3/blood) impact on hemodynamics of overlapped stenotic artery. Journal of Molecular Liquids, 248, 809-821.

[20] Ijaz, S., \& Nadeem, S. (2017). A biomedical solicitation examination of nanoparticles as drug agents to minimize the hemodynamics of a stenotic channel. The European Physical Journal Plus, 132(11), 448.

[21] Zaman, A., Ali, N., \& Ali, I. (2018). Effects of nanoparticles (Cu (Copper), Silver (Ag)) and slip on unsteady blood flow through a curved stenosed channel with aneurysm. Thermal Science and Engineering Progress, 5, 482-491.

[22] Zaman, A., Ali, N., \& Khan, A. A. (2020). Computational biomedical simulations of hybrid nanoparticles on unsteady blood hemodynamics in a stenotic artery. Mathematics and Computers in Simulation, 169, 117-132.

[23] Ellahi, R., Raza, M., \& Vafai, K. (2012). Series solutions of non-Newtonian nanofluids with Reynolds' model and Vogel's model by means of the homotopy analysis method. Mathematical and Computer Modelling, 55(7-8), 1876-1891.

[24] Ahmed, A., Nadeem, S. (2017). Effects of magnetohydrodynamics and hybrid nanoparticles on a micropolar fluid with 6-types of stenosis. Results in Physics, 7, 4130-4139. 
[25] Zaman, A., Ali, N., \& Khan, A. A. (2020). Computational biomedical simulations of hybrid nanoparticles on unsteady blood hemodynamics in a stenotic artery. Mathematics and Computers in Simulation, 169, 117-132.

[26] Devi, S. A., \& Devi, S. S. U. (2016). Numerical investigation of hydromagnetic hybrid CuAl2O3/water nanofluid flow over a permeable stretching sheet with suction. International Journal of Nonlinear Sciences and Numerical Simulation, 17(5), 249-257.

[27] A.C. Burton, Physiology and Biophysics of the Circulation, Introductory Text, YearBook Medical Publisher, Chicago, 1966.

[28] Hoffman, K. A., Chiang, S. T. (2000). Computational Fluid Dynamics. Engineering Education System, Kansas, 1, 486.

[29] Bég, O. Anwar, N. Ali, A. Zaman, Eemaan T. A. Bég and Ayesha Sohail, Computational modelling of heat transfer in annular porous medium solar energy absorber with a P1-radiative differential approximation, J. Taiwan Inst. Chemical Eng., 66, 258-268 (2016).

[30] Bég, O. Anwar, A. Zaman, N. Ali, S.A. Gaffar and T. A. Bég, Numerical computation of oscillatory two-immiscible magnetohydrodynamic flow in a dual porous media system: FTCS and FEM study, Heat Transfer 1-19 (2019). DOI: 10.1002/htj.21429

[31] A. Zaman, N. Ali and Bég, O. Anwar, Numerical study of unsteady blood flow through a vessel using Sisko model, Engineering Science and Technology-An International Journal, 19, 538-547 (2016).

[32] N. Ali, K. Javid, M. Sajid and Bég, O. Anwar, Numerical simulation of peristaltic flow of a biorheological fluid with shear-dependent viscosity in a curved channel, Computer Methods in Biomechanics and Biomedical Engineering, 19 (6) 614-627 (2016).

[33] A. Zaman, N. Ali, Bég, O. Anwar and M. Sajid, Heat and mass transfer to blood flowing through a tapered overlapping stenosed artery, Int. J. Heat Mass Transfer, 95, 1084-1095 (2015).

[34] N. Ali, A. Zaman, Bég, O. Anwar, T. Hayat, Mathematical model for isothermal wire-coating from a bath of Giesekus viscoelastic fluid, Chem. Eng. Comm. 203, 1336-1248 (2016).

[35] O.K. Baskurt, M.R. Hardeman, M.W. Rampling and H.J. Meiselman (Editors) Handbook of Hemorheology and Hemodynamics, Volume 69, Biomedical and Health Research: 468 pp, IOS Press, USA (2007). 
[36] S.K. Das, S.U.S. Choi, W. Yu and T. Pradeep, Nanofluids: Science and Technology, Wiley, USA (2007).

[37] Rytting E, Nguyen J, Wang X, et al. Biodegradable polymeric nanocarriers for pulmonary drug delivery. Expert Opin. Drug Deliv 5, 629-639 (2008).

[38] J. Prakash, D. Tripathi, Bég, O. Anwar, Comparative study of hybrid nanofluid performance in microchannel slip flow induced by electroosmosis and peristalsis, Applied Nanoscience (2020). (14 pages). doi.org/10.1007/s13204-020-01286-1 